\title{
Cooperative regulation of PBI1 and MAPKs precisely controls the master transcription factor WRKY45 in rice immunity
}

\section{Kota Ichimaru \\ Kindai University \\ Kenichi Harada \\ Osaka University \\ Shusuke Shigeta \\ Kindai University \\ Koji Yamaguchi \\ Kinki University \\ Keita Shimada \\ Kindai University}

\section{Kazuya Ishikawa}

Iwate Biotechnology Research Center

Kento Inoue

Kindai University

Yusaku Nishio

Kindai University

Satomi Yoshimura

Kindai University

Haruhiko Inoue

Institute of Agrobiological Sciences, National Agriculture and Food Research Organization (NARO)

Eiki Yamashita

Osaka University https://orcid.org/0000-0002-4278-0039

\section{Toshimichi Fujiwara}

Osaka University https://orcid.org/0000-0001-7739-3525

\section{Atsushi Nakagawa}

Osaka University https://orcid.org/0000-0002-1700-7861

\section{Chojiro Kojima}

Osaka University https://orcid.org/0000-0003-2723-8249

Tsutomu Kawasaki ( $\nabla$ t-kawasaki@nara.kindai.ac.jp )

Kindai University https://orcid.org/0000-0003-2579-2000 
Article

Keywords: Ubiquitination, protein degradation, pattern triggered immunity, rice, MAP kinase

Posted Date: September 9th, 2020

DOI: https://doi.org/10.21203/rs.3.rs-62065/v1

License: (c) (i) This work is licensed under a Creative Commons Attribution 4.0 International License. Read Full License

Version of Record: A version of this preprint was published at Nature Communications on May 16th, 2022. See the published version at https://doi.org/10.1038/s41467-022-30131-y. 
1 Cooperative regulation of PBI1 and MAPKs precisely controls the master transcription factor WRKY45 in rice immunity

Kota Ichimaru ${ }^{1 *}$, Kenichi Harada ${ }^{2 *}$, Shusuke Shigeta ${ }^{1 *}$, Koji Yamaguchi ${ }^{1 *}$, Keita Shimada $^{1 *}$, Kazuya Ishikawa ${ }^{1 \dagger}$, Kento Inoue ${ }^{1}$, Yusaku Nishio', Satomi Yoshimura ${ }^{1}$, Haruhiko Inoue ${ }^{3}$, Eiki Yamashita ${ }^{2}$, Toshimichi Fujiwara², Atsushi Nakagawa², Chojiro $K^{K o j i m a}{ }^{2,4, \ddagger}$ and Tsutomu Kawasaki ${ }^{1,5, \ddagger}$

'Department of Advanced Bioscience, Graduate School of Agriculture, Kindai University, Nakamachi, Nara 631-8505, Japan.

2Institute for Protein Research, Osaka University, 3-2 Yamadaoka, Suita, Osaka 5650871, Japan.

${ }^{3}$ Plant Function Research Unit, Division of Plant and Microbial Sciences, Institute of Agrobiological Sciences, National Agriculture and Food Research Organization (NARO), Kannondai 2-1-2, Tsukuba, Ibaraki, 305-8602, Japan.

${ }^{4}$ Graduate School of Engineering, Yokohama National University, 79-5 Tokiwadai, Hodogaya-ku, Yokohama, 240-8501, Japan.

${ }^{5}$ Agricultural Technology and Innovation Research Institute, Kindai University, Nakamachi, Nara 631-8505, Japan.

${ }^{\dagger}$ Present address: Iwate Biotechnology Research Center, Kitakami, Iwate 024-0003, Japan

${ }^{*}$ These authors contributed equally to this work.

${ }^{\ddagger}$ Correspondence

Tsutomu Kawasaki

E-mail: t-kawasaki@nara.kindai.ac.jp

Chojiro Kojima

E-mail: kojima-chojiro-xk@ynu.ac.jp. 


\section{SUMMARY}

2

3 The U-box type ubiquitin ligase PUB44 is targeted by the Xanthomonas oryzae XopP

4 effector and positively regulates pattern-triggered immunity in rice. Here we identified

5 PBI1, a protein that interacts with PUB44. Crystal structure analysis indicated that PBI1

6 forms a four-helix bundle structure. PBI1 also interacts with WRKY45, a master

7 transcriptional activator of rice immunity, and negatively regulates its activity. $\mathrm{PBI} 1$ is

8 degraded during the chitin response, and this is suppressed by silencing of PUB44 or

9 expression of $X o p P$, indicating that PBI1 degradation depends on PUB44. These data suggest that PBI1 suppresses WRKY45 activity when cells are in the unelicited state,

11 and during chitin signaling, PUB44-mediated degradation of PBI1 leads to activation of

12 WRKY45. In addition, phosphorylation of WRKY45 by MAP kinases releases WRKY45

13 from the PBI1-mediated inhibitory effect. These results demonstrate that chitin-induced

14 activation of WRKY45 is regulated by the cooperation between MAP kinase-mediated

15 phosphorylation and PUB44-mediated PBI1 degradation.

17 KEYWORDS:

18 Ubiquitination, protein degradation, pattern triggered immunity, rice, MAP kinase, 


\section{INTRODUCTION}

Plants have developed sophisticated immune systems to defend against pathogens and to restrict pathogen proliferation. Immune responses are initiated when plasma membrane-localized pattern-recognition receptors (PRRs) are activated by microbe-associated molecular patterns ${ }^{1}$. PRRs are receptor-like kinases with cytoplasmic kinase domains or receptor-like proteins with short cytoplasmic tails. Receptor-like kinases and receptor-like proteins possess extracellular ectodomains that directly bind to their ligands. Ligand perception by PRRs rapidly activates intracellular mitogen-activated protein (MAP) kinases and the production of reactive oxygen species ${ }^{2-}$ 5. These then activate a series of immune responses, including the production of antimicrobial compounds and reinforcement of the plant cell wall ${ }^{6}$. This kind of immunity is referred to as pattern-triggered immunity. To inhibit these host immune responses, pathogens deliver a variety of effectors into host cells ${ }^{7}$. The effectors target important host immune factors, including PRRs and downstream signaling factors, and suppress their activity, allowing the pathogens to colonize the plant. To overcome effectormediated inhibition of host immunity, plants have developed an effector-induced immune system referred to as effector-triggered immunity. This is mediated by intracellular immune receptors that are members of the nucleotide-binding leucine-rich repeat (NBLRR) protein family. These proteins are structurally similar to the NB oligomerization domain-like receptors (NLRs) of mammals ${ }^{1}$, and therefore, they are commonly referred to as plant NLRs ${ }^{8}$.

Chitin and peptidoglycan are typical microbe-associated molecular patterns derived from fungal and bacterial cell walls, respectively. In rice, chitin and peptidoglycan are recognized by the lysin motif (LysM) receptor-like proteins CEBiP and LYP4/6, 
1 espectively ${ }^{9-11}$. Upon ligand perception, CEBiP and LYP4/6 interact with the LysM

2 receptor-like kinase OsCERK1 at the plasma membrane, and then OsCERK1 transmits

3 the signal to intracellular components ${ }^{11,12}$. Thus, OsCERK1 is a common factor regulating

4 both fungal chitin- and bacterial peptidoglycan-triggered immunity. In response to the

5 chitin signal, the cytoplasmic kinase domain of OsCERK1 phosphorylates the rice

6 receptor-like cytoplasmic kinase OsRLCK185, which then phosphorylates MAP kinase

7 kinase kinases such as MAPKKK11, MAPKKK18, and MAPKKK24/MAPKKKع. These

8 kinases then trigger the intracellular activation of the MAP kinases MPK3 and MPK6 $6^{13-15}$.

9 The activated MAP kinases (MAPKs) induce robust immune responses by phosphorylating downstream immune factors including transcription factors.

Robust immune responses are mediated via the transcriptional regulation of immune-related genes by different types of transcription factors. The WRKY transcription factors are one of the transcription factor families that play important roles in plant

14 immunity ${ }^{16}$. WRKY45 is a major transcriptional activator of the immune response in rice.

15 Enhanced expression of WRKY45 confers resistance to bacterial blight caused by 16 Xanthomonas oryzae pv. oryzae (Xoo) and fungal blast diseases caused by 17 Magnaporthe oryzae ${ }^{17}$. The level of WRKY45 protein is regulated by the ubiquitinproteasome system ${ }^{18}$, and its activity is regulated via phosphorylation by the MAPK MPK6 ${ }^{19}$. In fact, the phosphomimic mutant of WRKY45 possesses enhanced transcriptional activity. In tobacco and Arabidopsis, the DNA binding activities of WRKYs are regulated via MAPK-mediated phosphorylation $20,21$.

WRKY45 has also been identified as a key regulator that participates in durable, broad-spectrum blast resistance mediated by the Panicle blast 1 (Pb1) gene. Pb1

24 encodes an NB-LRR protein with an N-terminal coiled coil (CC) domain (CC-NB-LRR) 22. 
$1 \quad \mathrm{~Pb} 1$ interacts with and stabilizes WRKY45 through its CC domain, probably by inhibiting

2 proteasome-mediated degradation of WRKY45, and this enhances the immune responses mediated by WRKY45.

WRKY45 appears to be autoregulated, because artificial expression of WRKY45 induces expression of the endogenous WRKY45 gene ${ }^{23}$. Since elevated levels of WRKY45 mRNA cause reductions in growth ${ }^{24,25}$, the transcriptional activity of WRKY45 must be suppressed in the absence of pathogens. This suggests the existence of a mechanism that inhibits WRKY45 transcriptional activity under unelicited condition.

Protein ubiquitination is an important post-translational modification process that marks target proteins for degradation via the proteasome ${ }^{26}$. Increasing evidence indicates that ubiquitination plays important roles in a variety of plant cellular processes including immunity, hormone responses, and development ${ }^{27}$. The ubiquitination reaction directs the covalent conjugation of conserved ubiquitin molecules onto protein substrates through the sequential activities of a ubiquitin-activating enzyme (E1), a ubiquitinconjugating enzyme (E2), and a ubiquitin ligase (E3). Plant E3 ligases are classified into three classes: HECT (homologous to E6-associated protein C-terminus), RING finger, and U- box ${ }^{26}$.

Recent studies have revealed that plant U-box type ubiquitin ligases (plant $\underline{\mathrm{U}}-$ box proteins; PUBs) are involved in the positive and negative regulation of defense responses against a variety of pathogens ${ }^{28}$. The tobacco PUB protein CMPG1 and its wheat homolog contribute positively to immune responses ${ }^{29,30}$. In contrast, the three closely related Arabidopsis PUBs AtPUB22, AtPUB23, and AtPUB24 negatively regulate pattern-triggered immunity ${ }^{31}$. AtPUB22 ubiquitinates Exo70B2, a subunit of the exocyst complex that mediates vesicle tethering during exocytosis ${ }^{32}$. AtPUB12 and AtPUB13 
1 ubiquitinate the flagellin receptor FLS2, leading to its degradation and the attenuation of 2 signaling by flagellin, which is another microbe-associated molecular pattern ${ }^{33,34}$. 3 AtPUB25 and AtPUB26 also negatively regulate flagellin signaling by marking the FLS2associated receptor-like cytoplasmic kinase BIK1 for degradation ${ }^{35}$. AtPUB12 and AtPUB13 also interact with the LysM receptor-like kinases CERK1 and LYK5, respectively, to negatively regulate pattern-triggered immunity ${ }^{36,37}$. The rice PUB protein SPL11 is the closest homologue of AtPUB12 and AtPUB13, and it negatively regulates cell death in rice ${ }^{38}$. Thus, PUBs regulate multiple steps in plant immunity.

Xoo uses a type III secretion system to deliver effectors to the plant cell ${ }^{39}$. XopP, one of the Xoo type III effectors, targets the rice U-box type ubiquitin ligase PUB44 ${ }^{40}$ and inhibits its ubiquitin ligase activity by interacting with its U-box domain. Silencing of PUB44 suppresses peptidoglycan- and chitin-induced immunity and resistance to $X_{0 O}{ }^{40}$, indicating that PUB44 functions downstream of OsCERK1. However, PUB44 is not involved in the activation of MAPKs that is regulated by OsRLCK $185^{40}$. This suggests that PUB44 plays a positive role in an immune pathway that is independent of, or downstream of OsRLCK185.

To understand the molecular mechanism by which PUB44 regulates patterntriggered immunity, we screened for proteins that interacted with PUB44. We identified PBI1 (브는-Interacting 1), which belongs to a protein family with the DUF1110 domain. PBI1 exhibits a unique tertiary structure composed of a four-helix bundle. This structure is similar to those of the CC domains of CC-NB-LRR immune receptors. Chitin treatment induces PUB44 phosphorylation and the PUB44-dependent degradation of PBI1, suggesting that PUB44 may induce immune responses through the degradation of PBI1. PBI1 interacts with WRKY45 and negatively regulates its activity. Therefore, it 
1 appears that PBI1 keeps WRKY45 in an inactive state in the absence of pathogen attack, and that PUB44-mediated degradation of PBI1 activates immunity via the desuppression of WRKY45. Knockout mutations of $P B / 1$ increase the protein levels of WRKY45, possibly by releasing it from the PBI1-mediated inhibition of WRKY45 autoregulation. In addition, the degradation of PBI1 is greatly reduced in the mapkkk11/mapkkk18 double mutant, suggesting that the MAPK pathway also regulates the chitin-induced degradation of PBI1. Thus, it is likely that PUB44 and the MAPKs cooperatively regulate WRKY45 via PBI1 in rice immunity.

\section{RESULTS}

\section{PBI1 interacts with PUB44}

PUB44 has ubiquitin ligase activity and positively regulates pattern-triggered immunity ${ }^{40}$. This suggests that PUB44 controls immune responses through ubiquitination of immune factors that interact with its ARM domain. To identify substrates for PUB44, we screened proteins that interact with the ARM domain using a yeast two-hybrid system. We identified the product of gene Os01g0156300 (Rice Annotation Project Database) as a candidate, and named the gene $\underline{P} U \underline{B} 44-\underline{I n t e r a c t i n g}$ protein 1 (PB/1). BLAST database searches revealed that PBI1 contains a DUF1110 domain with unknown function. Rice contains three additional DUF1110-containing proteins (PBI2-PBI4), and a phylogenic analysis indicated that PBI1 exhibits highest similarity to PBI2 (72\% identity at the amino acid level) (Fig. 1a). PBI2 is located adjacent to $P B / 1$ on chromosome 1, with an interval of approximately $1 \mathrm{~kb}$ between the coding regions. 
1 and each member of the PBI family. PUB44 interacts with PBI1 and PBI2, but not with

2 PBI3 or PBI4 (Fig. 1b). Rice PUB45 and PUB46 are the closest homologs of PUB44, but

3 they do not have the PUB44-specific U-box sequence that is targeted by the XopP 4 effector $^{40}$. Our two hybrid analyses indicated that neither PBI1 nor PBI2 interacts with PUB45 or PUB46 (Fig. 1c).

To identify which domains of PUB44 bind to PBI1 and PBI2, we used three constructs containing the U-box and/or ARM domains, separated by a 101 aa linker domain (Fig. 1d). These constructs were designated as PUB44 ${ }^{1-452}$, PUB44 ${ }^{1-203}$, and PUB44 ${ }^{102-452}$. A two-hybrid experiment indicated that PBI1 interacts with PUB44 ${ }^{102-452}$ but not PUB44 ${ }^{1-203}$ (Fig. 1d). This result is consistent with the fact that PBI1 was isolated using the ARM domain. In contrast, PBI2 interacts with PUB44 ${ }^{1-203}$ but not PUB44 ${ }^{102-452}$, indicating that $\mathrm{PBI} 2$ interacts with the U-box domain. Thus, PBI1 and PBI2 interact with different domains of PUB44, even though they are highly homologous with one another. The fact that PBI1 (but not PBI2) interacts with the ARM domain suggests that PBI1 may be the substrate of PUB44. Therefore, we focused on PBI1 in further studies.

\section{Decreases in PBI1 levels depend on PUB44 in response to chitin}

The interaction between PBI1 and the ARM domain of PUB44 suggests that the protein level of PBI1 may be regulated via a ubiquitin-proteasome pathway. To determine the protein level of PBI1 during the chitin response, we used recombinant PBI1 protein to raise an antibody (a-PBI1) that specifically recognizes PBI1 (Extended Data Fig. 1a). We treated rice suspension-cultured cells with $(\mathrm{GlcNAc})_{7}$, a chitin oligomer with a degree of polymerization of 7 , and carried out immunoblot analysis with a-PBI1. The PBI1 protein levels decreased gradually after chitin treatment (Fig. 2a). We then used quantitative 
1 real-time PCR to analyze $P B / 1$ transcript levels, and found that $P B / 1$ expression was not

2 significantly altered after chitin treatment (Fig. 2b). Therefore, it is likely that the chitin-

3 induced reduction in PBI1 occurs at the protein level. In addition, treatment with the

4 proteasome inhibitor MG132 induces the accumulation of PBI1 (Fig. 2c), indicating that

5 the PBI1 protein concentration is regulated via proteasome-mediated protein

6 degradation.

$7 \quad$ To test the possibility that PUB44 may participate in the chitin-induced 8 degradation of PBI1, we used rice PUB44 RNAi cells ${ }^{39}$ in which the levels of PUB44

9 mRNA was greatly reduced. Unexpectedly, an immunoblot with $\alpha-P B I 1$ indicated that the PBI1 protein level was reduced in the PUB44 RNAi cells (Fig. 2d). Chitin treatment failed to further reduce the level of PBI1 in these cells (Fig. 2d), suggesting that PUB44 is involved in the chitin-induced degradation of PBI1. We also generated two knockout mutants of PUB44 using the CRISPR/Cas9 system. An 8-bp deletion in pub44-1 removed the translation initiation codon, and a 1 bp deletion in the U-box region in pub442 caused a nonsense mutation (Extended Data Fig. 1b). No PUB44 protein was 16 detectable in either of these mutants (Extended Data Fig. 1c), and PBI1 protein levels were greatly reduced in both mutants (Fig. 2e). Quantitative real-time PCR showed that the PBI1 transcript levels were also reduced in the pub44 mutants (Fig. 2f), suggesting that the expression of $P B / 1$ may be regulated downstream of $P U B 44$.

The Xoo XopP effector inhibits PUB44 activity by interacting with its U-box domain ${ }^{40}$, and over-expression of $X o p P$ in plant cells suppresses pattern-triggered immunity mediated by PUB44 ${ }^{40}$. We treated rice suspension cells that were overexpressing $\mathrm{XopP}(\mathrm{XopP}$-ox cells) with chitin and examined the levels of PBI1 protein.

24 Chitin-induced degradation of PBI1 was significantly suppressed in the XopP-ox cells 
1 (Fig. 2g), supporting the possibility that PBI1 degradation may be regulated by the

2 PUB44-mediated ubiquitination pathway.

3

\section{PBI1 is composed of a four-helix bundle}

Although PBI1 consists mainly of the DUF1110 domain, the molecular nature of this domain was unknown. To elucidate the structure of PBI1, we used an E. coli protein expression system ${ }^{41}$, purified the recombinant PBI1 protein, and determined its tertiary structure. The crystal structure was solved at a resolution of $1.84 \AA$. PBI1 is composed of a four-helix bundle (Fig. 3a, b) with a diameter of approximately $19 \AA$ and a length of about $70 \AA$. There are six molecules in the asymmetric unit. The root mean square differences (r.m.s.d.) between each monomer are from 0.15 to $0.85 \AA$. We observed no large conformational change induced by crystal packing. The calculated solvent content was $63 \%$ (Matthews coefficient $=3.32 \AA^{3} \mathrm{Da}^{-1}$ ). The four helices of PBI1 are arranged in an up-down-up-down topology, and the bundle is leftward turning. The four helices are part of a single polypeptide chain (Ala10-His39, Glu49-Gly90, Leu110-Asp148, and Val110-Val191) and are connected to each other by three loops (Leu40-Asp48, Gly91Tyr109, and His149-Cys154). The interfaces between the helices consist of hydrophobic residues, whereas hydrophilic residues are exposed on the surfaces that interact with the aqueous environment. The hydrophobic residues occur as repeats of 3 or 4 residues per helical turn and form the core of the bundle structure.

We used the Dali server ${ }^{42}$ to perform a database search for three-dimensional structures that exhibit similarity to PBI1, and identified eight unique proteins with Zscores higher than 10. Seven of these proteins are: Methyl-accepting chemotaxis transducer ${ }^{43}, \mathrm{SH} 2$ domain $^{44}$, Talin $1^{45}$, surface protein VSPA ${ }^{46}$, focal adhesion kinase $1^{47}$, 
1 tyrosine kinase 2 beta $^{48}$, and superoxide dismutase ${ }^{49}$. In addition, the four-helix bundle

2 structure occurs in the CC domains of plant CC-NB-LRRs including Rx and MLA10 $10^{50-52}$.

3 In particular, the CC domain of Rx is very similar in structure to PBI1, with a high Z-score

4 of 4.5. A structural alignment of the Rx CC domain and PBI1 showed a high degree of

$5 \quad$ similarity (Extended Data Fig. 2).

6

$7 \quad$ PBI1 interacts with WRKY45 in the nucleus

$8 \quad$ To analyze the subcellular localization of PBI1 we made constructs encoding

9 green fluorescent protein (GFP) fused to the N- or C-terminal of PBI1. These constructs, along with one encoding red fluorescent protein (RFP) containing a nuclear localization signal (RFP-nls), were used to transfect rice protoplasts. Fluorescence from both the GFP-PBI1 and PBI1-GFP hybrid proteins was detected predominantly in the nuclei (Fig. 3c), although some GFP fluorescence was also observed in the cytoplasm. The presence of PBI1 in the nucleus suggests that it may be involved in transcriptional regulation. We screened for rice factors that interact with PBI1 and identified WRKY45 as a candidate. WRKY45 is a key regulator of rice immunity against rice blast and bacterial blight diseases ${ }^{24}$. To analyze the interaction between PBI1 and WRKY45, we performed a bimolecular fluorescence complementation (BiFC) assay using rice protoplasts. WRKY45 was tagged with the N-terminal domain of the yellow fluorescent protein Venus (WRKY45-Vn), and PBI1 was tagged with the C-terminal domain of Venus (PBI1-Vc). Transient expression of these constructs together resulted in fluorescence in the nucleus (Fig. 4a). We also examined the interaction between PBI1 and WRKY45 in a co-immunoprecipitation assay using rice protoplasts transiently expressing GFP-PBI1 and Myc-tagged WRKY45. Myc-WRKY45 co-immunoprecipitated 
1 with GFP-PBI1 (Fig. 4b), confirming the in vivo interaction between PBI1 and WRKY45.

The fact that WRKY45 interacts with PBI1 raises the possibility that WRKY45 may play a role in chitin-induced immunity. However, the involvement of WRKY45 in pattern-triggered immunity has not been described thus far. Our quantitative real-time PCR experiments demonstrated that expression of WRKY45 is activated after treatment with chitin (Fig. 4c). We also found that chitin-induced expression of WRKY62, which functions downstream of WRKY $45^{53}$, was significantly suppressed in two WRKY45knockdown lines (Fig. 4d and Extended Data Fig. 3a) ${ }^{53}$. These data indicate that WRKY45 participates in chitin-induced immunity.

The interaction between PBI1 and WRKY45 in nuclei suggests that PBI1 may be involved in the regulation of transcription by WRKY45. We carried out transactivation assays using effector constructs expressing Myc-tagged WRKY45 and PBI1, and a reporter construct containing a promoter with four W-box sequences upstream of the luciferase $\mathrm{cDNA}^{24}$. We transfected rice protoplasts with the Myc-tagged WRKY45 construct and the reporter, with or without the PBI1 construct, and examined the luciferase activity. Luciferase activity was increased in the presence of WRKY45 (Fig 4e and Extended Data Fig. 3b) but was significantly inhibited by co-expression of PBI1. This result indicates that PBI1 negatively regulates the transcriptional activity of WRKY45.

\section{pbi1 mutations cause increases in the protein levels of WRKY45.}

To clarify the roles of PBI1 in rice immunity, we generated two PBI1 knock-out mutants (pbi1-1 and pbi1-2) using the CRISPR/Cas9 system. pbi1-1 has a frameshift mutation caused by a 2-bp deletion, and pbi1-2 has a 6-bp deletion causing the loss of two aa residues and a 1-bp substitution within the PB/1-coding region (Extended Data 
1 Fig. 3c). Immunoblots with a-PBI1 showed that no PBI1 protein was detected in either mutant (Fig. 5a). Since no truncated PBI1-2 protein was detected in pbi1-2, the deletion of the two amino acid residues may have caused protein stability.

Both pbi1-1 and pbi1-2 exhibited a weak dwarf phenotype (Fig. 5b), which was similar to the phenotype of WRKY45-oxerexpressing plants ${ }^{24}$. Therefore, we analyzed the protein levels of WRKY45 by immunoblotting with $\alpha$-WRKY45. The WRKY45 protein levels were significantly increased in the pbi1 mutants compared with wild type (Fig. 5c). The WRKY45 transcript levels were also increased in the pbi1 mutants (Fig. 5d). Since WRKY45 is known to be autoregulated ${ }^{23}$, these results suggest that loss of PBI1 may result in the leaky autoactivation of WRKY45 transcription.

We examined the resistance of the pbi1 mutants to the compatible race $X_{0 o}$ T7174 by inoculating the plants using the clipping method. The pbi1 mutants developed disease lesions that were shorter than those of the wild type (Fig. 5e, f). Genomic quantitative PCR using specific primers for the $X$. oryzae XopA gene indicated that bacterial growth was also reduced in the pbi1 mutants (Fig. $5 \mathrm{~g}$ ). Thus, the pbi1 mutants enhanced resistance to $X$. oryzae, possibly via accumulation of WRKY45.

We also tested whether the pbi1 mutations affect chitin-induced expression of WRKY62 using cultured rice cells. Expression of WRKY62 was significantly enhanced in the pbi1 mutants (Fig. 5h). This is consistent with results of the transient transcription assay (Fig. 4e), in which WRKY45-mediated transcription was reduced in the presence of PBI1. This result also indicates that an additional component, apart from PBI1, functions as an activator of WRKY45, because chitin-induced expression of WRKY62 was observed in the absence of PBI1. 
1 Chitin-induced MAPK activation positively regulates PBI1 degradation.

Previously we reported that chitin perception triggers the phosphor-signaling pathway OsCERK1 - OsRLCK185 - MAPKKK11/MAPKKK18 - MKK4/MKK5 - MPK3/ MPK6 ${ }^{15}$. To ask whether this MAPK pathway affects chitin-induced degradation of PBI1, we produced mapkkk11/mapkkk18 double mutant lines by using the CRISPR-CAS9 system and the mapkkk11-1 mutant background, which was generated by a Tos 17 insertion in MAPKKK11 gene ${ }^{15}$. The mapkkk11-1/mapkkk18-1 and mapkkk111/mapkkk18-2 lines carry nonsense mutations caused by 1 bp insertions in MAPKKK18 gene (Extended Data Fig. 4a). Chitin-induced activation of MPK3 and MPK6 was significantly reduced in the mutants (Fig. 6a). As shown in Fig. 6b, chitin-induced PBI1 degradation was suppressed in the mapkkk11/mapkkk18 mutants, indicating that MAPK activity is required for the PBI1 degradation.

WRKY-type transcription factors are known to be activated by MAPK-mediated phosphorylation ${ }^{20}$. The three amino acid residues Thr266, Ser294, and Ser299, located in the C-terminal region of WRKY45, are phosphorylated by MPK6 ${ }^{19}$. The phosphorylation of Ser294 and Ser299 positively regulates the immune response, whereas a phosphor-mimic mutation of Thr266 inhibits immunity. To examine whether the phosphorylation of WRKY45 by MAPKs may affect the interaction between WRKY45 and PBI1, we analyzed the interaction using a split nano-luciferase assay. For this assay, the full length, N-terminal or C-terminal regions of WRKY45 were fused to the Small BIT (SmBiT) of NanoLuc, and PBI1 was fused to the Large BiT (LgBiT). The resultant constructs were used to transfect rice protoplasts, and then total luciferase activities were measured. The experiments indicated that PBI1 interacts more strongly with the Cterminal region of WRKY45 than with the N-terminal region (Fig. 6c). We produced a 
1 phosphor-mimic mutant (WRKY45 ${ }^{\mathrm{DD}}$ ) of WRKY45 in which Ser294 and Ser299 were

2 each substituted with Asp. The phosphor-mimic mutation suppressed the interaction

3 between WRKY45 and PBI1 (Fig. 6d). In addition, we tested the effect of the dominant-

4 active phosphor-mimic mutant $\left(\mathrm{MKK} 4^{\mathrm{DD}}\right)$ of $\mathrm{MKK} 4$, because expression of $\mathrm{MKK} 4^{\mathrm{DD}}$

$5 \quad$ induces activation of MPK3 and MPK6 ${ }^{54}$. The interaction between PBI1 and WRKY45

6 was also suppressed by co-expression with MKK4 $4^{\mathrm{DD}}$ (Fig. 6d). Our data indicated that

7 the phosphorylation of WRKY45 reduced the binding affinity between PBI1 and WRKY45.

8 It is possible that this reduced binding affinity may stimulate the PUB44-mediated

9 degradation of PBI1. In fact, chitin-induced expression of WRKY62 was strongly reduced in the mapkkk11/mapkkk18 mutants (Fig. 6e), whereas expression of WRKY45 was less affected by these mutations. These results suggest that the MAPK-mediated phosphorylation of WRKY45 and the PUB44-mediated degradation of PBI1 function cooperatively in the activation of WRKY45. immunoblots as shown by an arrow in Fig. 7a. The shifted band of PUB44 was detected by treatment with chitin. However, the shifted band was undetectable in the Oscerk $1^{11}$, and it disappeared after treatment with $\lambda$ phosphatase (Fig. 7b). These results indicate that PUB44 is phosphorylated in an OsCERK1-dependent manner. The phosphorylation of PUB44 was delayed and reduced in the mapkkk11/mapkkk18 mutant (Fig. 7c). In addition, the transcript level of OsCERK1 in the mapkkk11/18 mutant was lower than in wild type cells (Fig. $7 d$ ), suggesting that the steady-state levels of OsCERK1 transcript are controlled through the MAPK pathway. The reduced levels of OsCERK1 transcript in the mapkkk11/18 mutant may be the reason for the reduction and delay in PUB44 24 phosphorylation. Thus, it is possible that the defects in PBI1 degradation in the 
mapkkk11/mapkkk18 mutants is partially associated with the reduction of PUB44 phosphorylation.

\section{DISCUSSION}

PUB44 was originally identified as the target for $X$. oryzae type III effector XopP. Previous study indicated that PUB44 plays an important role in immune activation in response to bacterial peptidoglycan as well as fungal chitin. In rice, upon perception of peptidoglycan and chitin, the corresponding PRRs transmit the immune signals into intracellular components through OsCERK $1^{9-11}$. Therefore, PUB44 is most likely activated downstream of OsCERK1. However, the molecular mechanisms of how PUB44 is activated and how it regulates the downstream immune responses had been unknown so far. In this study, we found that upon perception of chitin, PUB44 is phosphorylated in an OsCERK1-dependent manner. We also identified PBI1 as an interactor with PUB44. During the chitin response, PBI1 is degraded in a PUB44-dependent manner, suggesting that PUB44 may control immunity through degradation of PBI1. In addition, PBI1 interacts with and inhibits WRKY45, a key regulator of rice immunity. PBI1 degradation is also regulated by MAPKs. The data presented here demonstrate that the chitininduced activation of WRKY45 is regulated by both MAPK-mediated phosphorylation and PUB44-mediated PBI1 degradation.

PBI1 is a novel protein carrying the DUF1110 domain, and it forms a small protein family with $\mathrm{PBI} 2, \mathrm{PBI} 3$, and PBI4. The biological function of this family has not been elucidated so far. In this study, we determined the crystal structure of PBI1 and found that it forms a four-helix bundle. Many other proteins have four-helix bundle 
1 structures, including the CC domains of the CC-NB-LRR-type immune receptors ${ }^{50,52}$. In

2 fact, the tertiary structure of PBI1 is very similar to that of the CC domain of Rx, which is 3 a CC-NB-LRR receptor. Interestingly, it has been reported that WRKY45 interacts with 4 the $\mathrm{CC}$ domain of $\mathrm{Pb} 1$, a CC-NB-LRR protein involved in rice blast resistance ${ }^{22} . \mathrm{Pb} 1$ is 5 predicted to positively regulate the abundance of WRKY45 protein by protecting it from 6 degradation by the ubiquitin proteasome system, however, the molecular mechanisms 7 have not been elucidated in detail. In contrast to Pb1, PBl1 appears to negatively 8 regulate the abundance of WRKY45 protein, because WRKY45 protein levels are higher $9 \quad$ in the pbi1 mutants than in the wild type.

The plant PUB family regulates a variety of biological responses, but the mechanisms of PUB activation remain largely unknown. In Arabidopsis, the activation of PUB22 is regulated by MAPK-mediated phosphorylation ${ }^{55}$. In this study, we found that PUB44 is phosphorylated in an OsCERK1-dependent manner upon chitin perception. The phosphorylation of PUB44 was also observed in the mapkkk11/mapkkk18 mutants, but it was delayed and reduced. Therefore, it is unlikely that MAPKs phosphorylate PUB44. The reduced level of phosphorylation may be explained by the fact that OsCERK1 expression was reduced in the mapkkk11/mapkkk18 mutants. The identification of protein kinases that phosphorylate PUB44 will be required for a further understanding of PUB44 activation.

The co-expression of PBI1 and WRKY45 in rice protoplasts indicated that PBI1 inhibits the transcriptional activity of WRKY45. Therefore, it is likely that PBI1 functions as a negative regulator of WRKY45 by direct interaction. In fact, the pbi1 plants contained increased levels of WRKY45 protein, possibly because of the leaky auto-activation of WRKY45 transcription. These increased levels of WRKY45 resulted in enhanced 
1 resistance to $\mathrm{Xoo}$, which is consistent with previous observations that overexpression of

$2 \quad W R K Y 45$ enhanced rice immunity ${ }^{17}$. On the other hand, the enhanced levels of WRKY45

3 mRNAs negatively affect plant growth ${ }^{24,25}$. Therefore, it is possible that negative

4 regulation of the WRKY45 transcriptional activity via PBI1 under unelicited condition is

$5 \quad$ important for growth and reproduction.

6

PBI1 is degraded upon chitin perception, and this is suppressed by silencing of

7 the PUB44 gene or expression of XopP. Thus, it is possible that PBI1 degradation occurs

8 via PUB44-mediated ubiquitination of PBI1. However, the ligase activity of full-length

9 PUB44 is very weak ${ }^{40}$, and we failed to detect ubiquitination of PBI1 by PUB44 in multiple in vitro ubiquitination assays. On the other hand, treatment of rice cells with the proteasome inhibitor MG132 resulted in the accumulation of PBI1, suggesting that PBI1 protein levels are likely regulated by the ubiquitin-proteasome pathway.

PBI1 inhibits the activity of WRKY45. Therefore, it is possible that the chitininduced degradation of PBI1 releases WRKY45 and activates WRKY45-mediated transcription. If the activation of WRKY45 is regulated only via PBI1 degradation, then WRKY45-mediated transcription would not occur in the absence of PBI1. However, chitin-induced expression of WRKY62 was still observed in the pbi1 mutants, indicating the existence of a positive regulatory mechanism for WRKY45 activation. Previous studies indicated that WRKY45 activity is regulated through the phosphorylation of its Cterminal region by $M P K 6^{19}$. In fact, ectopic activation of MPK6 increases the transcriptional activity of WRKY $45^{19}$. Consistent with this, we found that the mapkkk11/mapkkk18 mutations greatly reducing the activation of MPK3 and MPK6 strongly suppressed the chitin-induced expression of WRKY62. These results indicate

24 that the MAPKs regulate the chitin-induced activation of WRKY45. It has been shown 
1 that the DNA-binding activity of WRKYs is regulated via MAPK-mediated

2 phosphorylation ${ }^{20}$, however, it hasn't yet been shown that MPK3 and MPK6 control

3 WRKY45 activity in a similar manner.

4

This study and previous reports indicate that WRKY45 is regulated by both MAPK-mediated phosphorylation and PBI1 degradation. In addition, we also revealed a connection between MAPK-mediated phosphorylation and PBI1 degradation. The phosphorylation of WRKY45 by MAPKs reduces the binding affinity between PBI1 and WRKY45, suggesting that phosphorylation may stimulate the release of WRKY45 from PBI1. Furthermore, PBI1 degradation was suppressed in the mapkkk11/mapkkk18 mutants. Thus, it is possible that the PUB44-mediated degradation of PBI1 may require the disassociation between PBI1 and WRKY45.

Our study has revealed two regulatory mechanisms for WRKY45 activation, with both positive and negative regulation (Extended Data Fig. 4b). Under unelicited conditions, PBI1 inhibits WRKY45 activation in order to maintain its basal activity. Upon chitin perception, the MAPK cascade is activated, and the MAPKs phosphorylate WRKY45. This stimulates the release of WRKY45 from PBI1. At the same time, PUB44 is phosphorylated and then PBI1 is degraded, possibly following the disassociation from WRKY45.

The perception of microbe-associated molecular patterns induces the rapid transcription of immune-related genes, which is important for effective inhibition of pathogen growth. The protein phosphorylation- and ubiquitination-based mechanisms that control the activities of transcription factors are likely able to induce expression of downstream genes much more rapidly than mechanisms involving the transcriptional control of genes encoding transcription factors. Therefore, it seems that the cooperative 
1 regulation of WRKY45 via both the PUB44-PBI1 and MAPK-pathways contributes to the

2 rapid activation of immunity in rice. Although WRKY45 is a key factor for the activation

3 of rice immunity, its enhanced activation negatively affects plant growth ${ }^{24,25}$. Therefore, 4 the strict regulation of WRKY45 may be required for balancing immunity and growth.

$7 \quad$ Methods

\section{$8 \quad$ Plant materials}

9 Rice (Oryza sativa) Japonicum cultivar Nipponbare was used as the wild type. The pbi1, pub44, and mapkkk18 mutants were generated using the CRISPR/Cas9 system as described below. The mutants mapkkk11 15, WRKY45-kd ${ }^{23}$, and PUB44-kd and XopP$O X^{40}$ were described previously.

\section{Plasmid constructs.}

Full length cDNAs for PBI1 (Os01g0156300), PBI2 (Os01g0156400), PBI3 (Os01g0157100), PBI4 (Os03g0373300), and WRKY45(Os05g0322900) were amplified by PCR from cDNAs prepared from wild-type rice leaves and ligated into the $p E N T R / D$ TOPO cloning vector. The plasmids containing PUB44, PUB45, and PUB46 were described previously ${ }^{40}$. The $P B / 1$ and WRKY45 cDNA fragments were transferred using the Gateway system with an LR clonase reaction into p35S-GFP-GW for the subcellular localization assays and into p35S-Vn-GW and p35S-Vc-GW for the BiFC assays ${ }^{13}$. For the two hybrid assays, DNA fragments of the PBI1-PBI4 and PUB44-PUB46 coding regions were transferred using the Gateway system with an LR clonase reaction into vectors pBTM116 (bait) and pVP16 (prey) ${ }^{56}$. 


\section{Plant transformation using CRISPR/Cas9}

3 The guide RNA cloning vector pU6gRNA and the all-in-one Cas9/gRNA vector 4 pZDgRNA_Cas9ver.2_HPT were kindly provided by Dr. Endo ${ }^{57}$. The 20 bp sequences 5 from +5 to +24 of PBI1 (CGGCGGAGGCGTGGAGATCG), from -19 to +1 of PUB44

6 (TCCCCACGGGGCAATCGCCA), from +155 to +174 of PUB44 7 (CGTACGACCGGGCGTCCATA), and from +337 to +356 of MAPKKK18 8 (ATCTCGAGGACCGCGAGTAA) were selected as the target sites of Cas9 by using the 9 CRISPR-P website (http://cbi.hzau.edu.cn/cgi-bin/CRISPR). These were cloned into pZDgRNA_Cas9ver.2_HPT ${ }^{57}$, and the constructs were introduced into embryogenic WT rice calli by Agrobacterium-mediated transformation ${ }^{58}$. To identify the knockout mutants of each gene, genomic DNA was extracted from hygromycin-resistant calli or regenerated plants. The genomic regions containing the Cas9 target sites were amplified by PCR and sequenced as previously described ${ }^{57}$.

\section{Rice transformation}

17 Calli generated from rice embryos were transformed using Agrobacterium tumefaciens

18 EHA101 lines carrying each construct, as described previously ${ }^{58}$. The transformed calli were selected by resistance to hygromycin and used for the generation of suspensioncultured cells.

\section{Yeast two-hybrid assays}

23 The yeast two-hybrid screening and interaction assays were based on the requirement

24 for histidine for yeast growth, as described previously ${ }^{40}$. 
2 Chitin treatments

3 Rice suspension-cultured cells were subcultured for 3 days in fresh medium, divided into 4 12-well plates (150 mg cells, $2 \mathrm{ml}$ fresh medium per well), and treated with $2 \mu \mathrm{g} / \mathrm{ml}$ $5 \quad(\mathrm{GlcNAc})_{7}$.

7 RNA isolation and quantitative real time PCR

8 Total RNA was isolated from rice suspension-cultured cells and leaves using TRIzol 9 reagent (Invitrogen) and then treated with RNase-free DNase I (Roche). First-strand cDNA was synthesized from $1 \mu \mathrm{g}$ total RNA with an oligo-dT primer and ReverTra Ace reverse transcriptase (Toyobo). Expression levels were quantified by quantitative real time PCR using the SYBR Green master mix (Applied Biosystems) in a Step-One Plus Real-Time PCR system (Applied Biosystems). The expression levels were normalized against a ubiquitin reference gene. Three biological replicates were used for each experiment, and two quantitative replicates were performed for each biological replicate.

\section{Protein extraction and immunoblotting}

Total protein was extracted in a buffer containing $100 \mathrm{mM}$ Tris- $\mathrm{HCl} \mathrm{pH} 7.5,20 \%(\mathrm{v} / \mathrm{v})$ glycerin, and a protease inhibitor cocktail (Roche), and analyzed by protein immunoblotting with $\alpha-P B I 1, \alpha-P U B 44{ }^{40}, \alpha-W R K Y 45^{22}$ or $\alpha$-pMAPK (Cell Signaling). Polyclonal antibodies against PBI1 (prepared by Medical Biological Laboratories) were raised in rabbits using the full length PBI1 protein as the antigen. For $\lambda$ phosphatase treatment, total protein was incubated with $\lambda$ phosphatase (Santa Cruz Biotechnology, $200312 \mathrm{~A}$ ) at $30^{\circ} \mathrm{C}$ for 90 mins and subsequently subjected to immunoblotting. 


\section{Co-immunoprecipitation}

3 Rice protoplasts transiently expressing GFP-PBI1 and Myc-tagged WRKY45 were 4 frozen in liquid nitrogen and resuspended in extraction buffer $(50 \mathrm{mM}$ Tris- $\mathrm{HCl}(\mathrm{pH} 7.5)$, $5150 \mathrm{mM} \mathrm{NaCl}, 10 \%$ glycerol, $5 \mathrm{mM}$ DTT, $2.5 \mathrm{mM} \mathrm{NaF}, 1.5 \mathrm{mM}$ Na3Vo4, 1x Complete 6 EDTA free protease inhibitor cocktail (Roche) and $2 \%(\mathrm{v} / \mathrm{v})$ IGEPAL CA-630 (MP 7 Biomedicals)). The supernatant was incubated with GFP-Trap beads (Chromotek). The

8 beads were washed four times with the extraction buffer and resuspended in an equal

9 volume of $2 \times$ SDS sample buffer. Co-immunoprecipitated proteins were analyzed by immunoblots with $\alpha-$ GFP (Abcam, ab6556) or a-Myc (Nakarai, 04362-34).

\section{Transient assays using rice protoplasts}

Protoplasts were isolated from cultured rice cells by digestion of the cell walls with

14 Cellulase RS (Yakult) and Macerozyme R-10 (Yakult) as described previously ${ }^{58}$. Aliquots $15(100 \mu \mathrm{l})$ of protoplasts $\left(2.5 \times 10^{6} \mathrm{cells} / \mathrm{ml}\right)$ were transformed with plasmid DNA using the 16 polyethylene glycol (PEG) method ${ }^{59}$. For the localization analysis and the BiFC assays, 17 transfected protoplasts were observed using a fluorescence microscope, the Axio Imager 18 M2 (Carl Zeiss) with the ApoTome2 system (Carl Zeiss). The transactivation assay of WRKY45 was carried out as described previously ${ }^{24}$. The reporter plasmid contained the firefly (F)-LUC gene downstream of a promoter containing $4 \times \mathrm{W}$-box sequences ${ }^{24}$. The Myc-tagged WRKY45 construct with the ubiquitin promoter 22 and the p35S-PBI1 construct were used as the effectors. The Renilla (R)-LUC gene under the control of the ubiquitin promoter was used as the internal control, and transcriptional activity was

24 measured as the ratio of LUC activities (F-LUC/R-LUC). 


\section{Crystallography}

3 The over-expression, purification, crystallization, and preliminary $\mathbf{X}$-ray analysis of native

4 and selenomethionine-labeled PBI1 were performed as described previously ${ }^{41}$. The

5 experimental phase and density modification were calculated from SAD data using

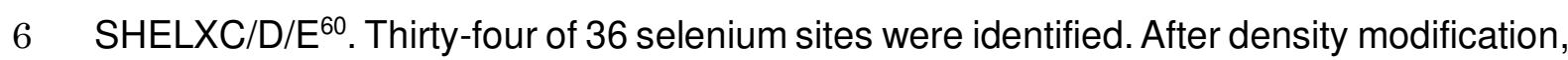

7 the figure of merit improved from 0.38 to 0.66 . Automatic model building was performed

8 using Buccaneer ${ }^{61}$. Further structure refinement was performed with $\operatorname{Coot}^{62}$ and

9 REFMAC5 $5^{63}$. The coordinates and structure factors have been deposited with the PDB

10 (http://pdbj.org) with accession code 7CJC. Data collection and refinement statistics are

11 given in Table S1. The structural model was evaluated using Rampage ${ }^{64}$.

\section{Split Nano Luciferase assay}

DNA fragments of PBI1, WRKY45 ${ }^{1-326}, W R K Y 45^{1-174}, W R K Y 45^{175-326}$, and WRKY45 were transferred using the Gateway system with LR clonase reactions into p35S-LgBiTT7-GW or p35S-SmBiT-T7-GW (K. Taoka, paper in preparation). The plasmid containing $M K K 4^{D D}$ was described previously ${ }^{13}$. The Firefly Luciferase gene under the control of CaMV 35S promoter was used as an internal control. The indicated combinations of plasmids were used to transfect rice protoplasts. After $18 \mathrm{~h}$ incubation at $30^{\circ} \mathrm{C}$, the activities of the Firefly and NanoLuc luciferases were measured on a TriStar2 LB942 luminometer (Berthold) using the ONE-Glo Luciferase Assay System (Promega) and the Nano-Glo Live Cell Assay System (Promega).

\section{Pathology Assays}


1 Fully expanded rice leaves were inoculated with a compatible race of bacterial blight

2 pathogen Xanthomonas oryzae pv. oryzae T7174 by clipping the leaf tips with scissors

3 that had been immersed in bacterial suspension (OD600 $=0.2)$. Symptoms were scored

4 by measuring lesion length 14 days after infection. The bacterial population of Xoo T7174

5 was also analyzed by quantitative real-time PCR. The DNA levels of the $X_{\circ o}$ XopA gene

6 relative to those of the rice ubiquitin gene were measured using genomic DNAs purified

7 from the infected leaves.

8

9 SUPPLEMENTAL INFORMATION

10 Extended Data include four figures and one table, and can be found with this article 11 online.

\section{AUTHOR CONTRIBUTIONS}

K.H., K.Y., C.K, and T. K. designed the project, analyzed the results and wrote the paper.

16 K.Ichimaru, S.S., K.S., K. Inoue, K.Ishikawa, Y.N., and S.Y. performed the experiments.

17 K.H., E.Y., T.F., A.N. and C.K. determined the crystal structure of PBI1. H.I. provided

18 materials. All authors discussed the results and commented on the manuscript.

\section{ACKNOWLEDGEMENTS}

We thank Jeff Dangl for critical reading of this manuscript, M. Endo for the CRISPR/Cas9 expression constructs, Y. Nishizawa for Oscerk1-ko mutant, K. Taoka for the split Nano

24 Luciferase assay constructs, S. Kitano for technical assistance, and members of the 
1 Kawasaki Laboratory for technical assistance and discussions. This research was

2 supported by Grants-in-Aid for Scientific Research (A)(19H00945), for Scientific

3 Research on Innovative Areas (18H04789), and Basic Science Research Projects from

4 the Mitsubishi Foundation to T.K.; by Grants-in-Aid for Scientific Research (JP15K18649)

5 and Basic Science Research Projects from the Sumitomo Foundation to K. Yamaguchi,

6 and by Grants-in-Aid for Scientific Research (B)(20H03191) and for Scientific Research

7 on Innovative Areas (19H04856) to C.K. Diffraction data were collected at the Osaka

8 University beamline BL44XU at SPring-8 (Harima, Japan) (Proposal No. 2015A6500).

9

10

\section{DECLARATION OF INTERESTS}

The authors declare no competing interests.

\section{References}

1 Dangl, J. L., Horvath, D. M. \& Staskawicz, B. J. Pivoting the plant immune system from dissection to deployment. Science 341, 746-751 (2013).

2 Meng, X. \& Zhang, S. MAPK cascades in plant disease resistance signaling. Annu. Rev. Phytopathol. 51, 245-266 (2013).

$3 \mathrm{Xu}$, J. \& Zhang, S. Mitogen-activated protein kinase cascades in signaling plant growth and development. Trends Plant Sci. 20, 56-64 (2015).

4 Kadota, Y. et al. Direct regulation of the NADPH oxidase RBOHD by the PRRassociated kinase BIK1 during plant immunity. Mol. Cell 54, 43-55 (2014).

5 Yamada, K. et al. The Arabidopsis CERK1-associated kinase PBL27 connects chitin perception to MAPK activation. EMBO J. 35, 2468-2483 (2016).

6 Malinovsky, F. G., Fangel, J. U. \& Willats, W. G. The role of the cell wall in plant immunity. Front. plant Sci. 5, (2014).

7 Dou, D. \& Zhou, J. M. Phytopathogen effectors subverting host immunity: 
different foes, similar battleground. Cell Host Microbe 12, 484-495 (2012).

8 Jones, J. D., Vance, R. E. \& Dangl, J. L. Intracellular innate immune surveillance devices in plants and animals. Science 354, aaf6395 (2016).

Kaku, H. et al. Plant cells recognize chitin fragments for defense signaling through a plasma membrane receptor. Proc. Natl. Acad. Sci. USA 103, 1108611091 (2006).

Liu, B. et al. Lysin motif-containing proteins LYP4 and LYP6 play dual roles in peptidoglycan and chitin perception in rice innate immunity. Plant Cell 24, 34063419 (2012).

11 Kouzai, Y. et al. Targeted gene disruption of OsCERK1 reveals its indispensable role in chitin perception and involvement in the peptidoglycan response and immunity in rice. Mol Plant Microbe Interact 27, 975-982 (2014).

12 Shimizu, T. et al. Two LysM receptor molecules, CEBiP and OsCERK1, cooperatively regulate chitin elicitor signaling in rice. Plant J. 64, 204-214 (2010).

13 Yamaguchi, K. et al. A receptor-like cytoplasmic kinase targeted by a plant pathogen effector is directly phosphorylated by the chitin receptor and mediates rice immunity. Cell Host Microbe 13, 347-357 (2013).

14 Wang, C. et al. OsCERK1-mediated chitin perception and immune signaling requires receptor-like cytoplasmic kinase 185 to activate an MAPK cascade in rice. Mol. Plant 10, 619-633 (2017).

15 Yamada, K., Yamaguchi, K., Yoshimura, S., Terauchi, A. \& Kawasaki, T. Conservation of chitin-induced MAPK signaling pathways in rice and Arabidopsis. Plant Cell Physiol. 58, 993-1002 (2017).

6 Tsuda, K. \& Somssich, I. E. Transcriptional networks in plant immunity. New Phytol. 206, 932-947 (2015).

17 Shimono, M. et al. Rice WRKY45 plays important roles in fungal and bacterial disease resistance. Mol. Plant Pathol. 13, 83-94 (2012).

18 Matsushita, A. et al. Nuclear ubiquitin proteasome degradation affects WRKY45 function in the rice defense program. Plant J. 73, 302-313 (2013).

19 Ueno, Y. et al. Abiotic stresses antagonize the rice defence pathway through the tyrosine-dephosphorylation of OsMPK6. PLoS Pathog. 11, e1005231 (2015). 
120 Ishihama, N., Yamada, R., Yoshioka, M., Katou, S. \& Yoshioka, H. 2 Phosphorylation of the Nicotiana benthamiana WRKY8 transcription factor by 3 MAPK functions in the defense response. Plant Cell 23, 1153-1170 (2011).

421 Mao, G. et al. Phosphorylation of a WRKY transcription factor by two pathogenresponsive MAPKs drives phytoalexin biosynthesis in Arabidopsis. Plant Cell23, 1639-1653 (2011).

22 Inoue, $\mathrm{H}$. et al. Blast resistance of CC-NB-LRR protein $\mathrm{Pb} 1$ is mediated by WRKY45 through protein-protein interaction. Proc. Natl. Acad. Sci. USA 110, 9577-9582 (2013).

23 Nakayama, A. et al. Genome-wide identification of WRKY45-regulated genes that mediate benzothiadiazole-induced defense responses in rice. BMC Plant Biol. 13, 150 (2013).

24 Shimono, M. et al. Rice WRKY45 plays a crucial role in benzothiadiazoleinducible blast resistance. Plant Cell 19, 2064-2076 (2007).

25 Wang, J. et al. A single transcription factor promotes both yield and immunity in rice. Science 361, 1026-1028 (2018).

26 Vierstra, R. D. The ubiquitin-26S proteasome system at the nexus of plant biology. Nat. Rev. Mol. Cell Biol. 10, 385-397 (2009).

27 Sadanandom, A., Bailey, M., Ewan, R., Lee, J. \& Nelis, S. The ubiquitinproteasome system: central modifier of plant signalling. New Phytol. 196, 13-28 (2012).

28 Trujillo, M. News from the PUB: plant U-box type E3 ubiquitin ligases. J. Exp. Bot. 69, 371-384 (2018).

29 Gonzalez-Lamothe, R. et al. The U-box protein CMPG1 is required for efficient activation of defense mechanisms triggered by multiple resistance genes in tobacco and tomato. Plant Cell 18, 1067-1083 (2006).

30 Zhu, Y. et al. E3 ubiquitin ligase gene CMPG1-V from Haynaldia villosa L. contributes to powdery mildew resistance in common wheat (Triticum aestivum L.). Plant J. 84, 154-168 (2015).

31 Trujillo, M., Ichimura, K., Casais, C. \& Shirasu, K. Negative regulation of PAMPtriggered immunity by an E3 ubiquitin ligase triplet in Arabidopsis. Curr. Biol. 18, 
1396-1401 (2008).

32 Stegmann, M. et al. The ubiquitin ligase PUB22 targets a subunit of the exocyst complex required for PAMP-triggered responses in Arabidopsis. Plant Cell 24, 4703-4716 (2012).

33 Lu, D. et al. Direct ubiquitination of pattern recognition receptor FLS2 attenuates plant innate immunity. Science 332, 1439-1442 (2011).

34 Zhou, J. et al. The dominant negative ARM domain uncovers multiple functions of PUB13 in Arabidopsis immunity, flowering, and senescence. J. Exp. Bot. 66, 3353-3366 (2015).

35 Wang, J. et al. A regulatory module controlling homeostasis of a plant immune kinase. Mol. Cell 69, 493-504 (2018).

36 Yamaguchi, K., Mezaki, H., Fujiwara, M., Hara, Y. \& Kawasaki, T. Arabidopsis ubiquitin ligase PUB12 interacts with and negatively regulates Chitin Elicitor Receptor Kinase 1 (CERK1). PLoS One 12, e0188886 (2017).

37 Liao, D. et al. Arabidopsis E3 ubiquitin ligase PLANT U-BOX13 (PUB13) regulates chitin receptor LYSIN MOTIF RECEPTOR KINASE5 (LYK5) protein abundance. New Phytol. 214, 1646-1656 (2017).

38 Fan, J. et al. The Monocot-Specific Receptor-like Kinase SDS2 Controls Cell Death and Immunity in Rice. Cell Host Microbe 23, 498-510 (2018).

39 Furutani, A. et al. Identification of novel type III secretion effectors in Xanthomonas oryzae pv. oryzae. Mol. Plant Microbe Interact. 22, 96-106 (2009).

40 Ishikawa, K. et al. Bacterial effector modulation of host E3 ligase activity suppresses PAMP-triggered immunity in rice. Nat. Commun. 5, 5430 (2014).

41 Harada, K. et al. Plant-specific DUF1110 protein from Oryza sativa: expression, purification and crystallization. Acta Crystallogr. Section F. 72, 480-484 (2016).

42 Holm, L. \& Rosenstrom, P. Dali server: conservation mapping in 3D. Nucleic Acids Res. 38, W545-549 (2010).

43 Pineda-Molina, E. et al. Evidence for chemoreceptors with bimodular ligandbinding regions harboring two signal-binding sites. Proc. Natl. Acad. Sci. USA 109, 18926-18931 (2012).

44 Mace, P. D. et al. NSP-Cas protein structures reveal a promiscuous interaction 
module in cell signaling. Nat. Struct. Mol. Biol. 18, 1381-1387 (2011).

45 Goult, B. T. et al. The domain structure of talin: residues 1815-1973 form a fivehelix bundle containing a cryptic vinculin-binding site. FEBS Lett. 584, 2237-2241 (2010).

46 Lawson, C. L., Yung, B. H., Barbour, A. G. \& Zuckert, W. R. Crystal structure of neurotropism-associated variable surface protein 1 (Vsp1) of Borrelia turicatae. J. Bacteriol. 188, 4522-4530 (2006).

47 Arold, S. T., Hoellerer, M. K. \& Noble, M. E. The structural basis of localization and signaling by the focal adhesion targeting domain. Structure 10, 319-327 (2002).

48 Lulo, J., Yuzawa, S. \& Schlessinger, J. Crystal structures of free and ligand-bound focal adhesion targeting domain of Pyk2. Biochem. Biophys. Res. Commun. 383, 347-352 (2009).

49 Wuerges, J. et al. Crystal structure of nickel-containing superoxide dismutase reveals another type of active site. Proc. Natl. Acad. Sci. USA 101, 8569-8574 (2004).

50 Maekawa, T. et al. Coiled-coil domain-dependent homodimerization of intracellular barley immune receptors defines a minimal functional module for triggering cell death. Cell Host Microbe 9, 187-199 (2011).

51 Hao, W., Collier, S. M., Moffett, P. \& Chai, J. Structural basis for the interaction between the potato virus $X$ resistance protein $(R x)$ and its cofactor Ran GTPaseactivating protein 2 (RanGAP2). J. Biol. Chem. 288, 35868-35876 (2013).

52 Casey, L. W. et al. The CC domain structure from the wheat stem rust resistance protein Sr33 challenges paradigms for dimerization in plant NLR proteins. Proc. Natl. Acad. Sci. USA 113, 12856-12861 (2016).

53 Fukushima, S., Mori, M., Sugano, S. \& Takatsuji, H. Transcription factor WRKY62 plays a role in pathogen defense and hypoxia-responsive gene expression in rice. Plant Cell Physiol. 57, 2541-2551 (2016).

54 Kishi-Kaboshi, M. et al. A rice fungal MAMP-responsive MAPK cascade regulates metabolic flow to antimicrobial metabolite synthesis. Plant J. 63, 599-612 (2010). 55 Furlan, G. et al. Changes in PUB22 Ubiquitination modes triggered by MITOGEN- 
ACTIVATED PROTEIN KINASE3 dampen the immune response. Plant Cell 29, 726-745 (2017).

56 Yamaguchi, K. et al. SWAP70 functions as a Rac/Rop guanine nucleotideexchange factor in rice. Plant J. 70, 389-397 (2012).

57 Mikami, M., Toki, S. \& Endo, M. Comparison of CRISPR/Cas9 expression constructs for efficient targeted mutagenesis in rice. Plant Mol. Biol. 88, 561-572 (2015).

58 Hiei, Y., Ohta, S., Komari, T. \& Kumashiro, T. Efficient transformation of rice (Oryza sativa L.) mediated by Agrobacterium and sequence analysis of the boundaries of the T-DNA. Plant J. 6, 271-282 (1994).

59 Kawano, Y. et al. Activation of a Rac GTPase by the NLR family disease resistance protein Pit plays a critical role in rice innate immunity. Cell Host Microbe 7, 362-375 (2010).

60 Chen, L. et al. The Hop/Sti1-Hsp90 chaperone complex facilitates the maturation and transport of a PAMP receptor in rice innate immunity. Cell Host Microbe 7, 185-196 (2010).

61 Uson, I. \& Sheldrick, G. M. Advances in direct methods for protein crystallography. Curr. Opin. Struct. Biol. 9, 643-648 (1999).

62 Cowtan, K. The Buccaneer software for automated model building. 1. Tracing protein chains. Acta Crystallogra. D Biol. Cryst. 62, 1002-1011 (2006).

63 Murshudov, G. N., Vagin, A. A. \& Dodson, E. J. Refinement of macromolecular structures by the maximum-likelihood method. Acta Crystallogra. D Biol. Cryst. 53, 240-255 (1997).

64 Lovell, S. C. et al. Structure validation by Calpha geometry: phi,psi and Cbeta deviation. Proteins 50, 437-450 (2003).

\section{FIGURE LEGENDS}

Fig. 1. Proteins containing the DUF1110 domain form a small protein family in rice.

a, Phylogenetic tree of the rice DUF1110 domain-containing proteins. Full-length protein sequences were used in the alignment. The neighbor-joining phylogenetic tree was 
1 created using ClustalW on the DNA Data Bank of Japan website

2 (http://www.ddbj.nig.ac.jp/). The tree was generated from the modified alignment using

3 Treeview $\mathrm{X}$ software. The percentages indicate amino acid identity with PBI1. b,

4 Interactions between PBI family members and PUB44 in yeast two-hybrid experiments.

5 Growth of yeast colonies on -ULWH plates (lacking uracil, leucine, tryptophan, and

6 histidine) indicates a positive interaction. c, Interactions of PBI1 and PBI2 with PUB44,

7 PUB45, and PUB46 in yeast two-hybrid experiments. Growth of yeast colonies on -

8 ULWH plates with $2 \mathrm{mM}$ 3-aminotriazole (3-AT) indicates a positive interaction. $\mathbf{d}$, (Upper

9 panel) Schematic diagram of PUB44 constructs. (Bottom panel) Interactions of PBI1 and PBI2 with each domain of PUB44 in yeast two-hybrid experiments. Positive interactions 11 are as for $(\mathbf{c})$.

Figure 2. Chitin-induced degradation of PBI1

a, Total proteins were prepared from wildtype (WT) rice suspension-cultured cells after treatment with $2 \mu \mathrm{g} / \mathrm{ml}$ (GluNAc) 7 and subjected to immunoblots with $\alpha-P B I 1 . \mathbf{b}, P B / 1$ transcript levels in rice cells treated with $2 \mu \mathrm{g} / \mathrm{ml}(\mathrm{GluNAc})_{7}$ were analyzed using quantitative real-time PCR. Data are means $\pm S D$ from three independent biological replicates, where each biological replicate consisted of two technical replicates. c, PBI1 protein levels in rice cells after treatment with the proteasome inhibitor MG132 (30 $\mu \mathrm{M})$ or dimethylsulphoxide (DMSO; mock), determined by immunoblot analysis with a-PBI1. d, PBI1 protein levels in PUB44 RNAi cells after treatment with $2 \mu \mathrm{g} / \mathrm{ml}$ (GluNAc) determined by immunoblot analysis with a-PBI1. e, PBI1 protein levels in the PUB44 knockout mutants, determined by immunoblot analysis with $\alpha$-PBI1. f, PB/1 transcript

24 levels in the PUB44 knockout mutants, analyzed using quantitative real-time PCR. Data 
1 are means $\pm S D$ from three independent biological replicates, where each biological

2 replicate consisted of two technical replicates. The asterisks indicate statistically 3 significant differences from the WT controls by Student's t-test $(P<0.05)$. g, PBI1 protein 4 levels in XopP-ox cells after treatment with $2 \mu \mathrm{g} / \mathrm{ml}$ (GluNAc) 7 , determined by $5 \quad$ immunoblot analysis with $\alpha-\mathrm{PBI} 1$.

$7 \quad$ Fig. 3. PBI1, with a four-helix bundle structure, localizes mainly to the nucleus.

8 a, Side view of PBI1, which forms a four-helix bundle. Coloring is from blue at the N-

9 terminus to red at the C-terminus. b, End view, with $\mathrm{N}$ - and $\mathrm{C}$-termini at the front. c, Detection of GFP-PBI1 and PBI1-GFP after transient expression in rice protoplasts. mCherry with a nuclear localization signal was used as a nuclear localization marker.

12 Scale bar=10 $10 \mathrm{~m}$.

Fig. 4. PBI1 interacts with and inhibits WRKY45

a, Bimolecular fluorescence complementation (BiFC) analysis was used to visualize the interaction between PBI1-Vc and WRKY45-Vn in rice protoplasts. mCherry with a nuclear localization signal was used as a nuclear localization marker. The $\beta$ glucuronidase (GUS) protein was used as a negative control. Scale bar $=10 \mu \mathrm{m} . \mathbf{b}$, Rice protoplasts were co-transfected with GFP-PBI1 and Myc-tagged WRKY45 and subjected to a co-immunoprecipitation assay. Proteins were precipitated using an antibody against GFP ( $\alpha$-GFP), and the input proteins and precipitated proteins were probed with $\alpha$-Myc and $\alpha-G F P . \quad$ c, WRKY45 transcript levels in rice suspension-cultured cells treated with $2 \mu \mathrm{g}(\mathrm{GluNAc})_{7}$ were analyzed using quantitative real-time PCR. d, Expression levels of WRKY62 in wild type and WRKY45-knockdown (kd) leaves after treatment with $2 \mu \mathrm{g} / \mathrm{ml}$ 
1 (GluNAc) $)_{7}$, analyzed using quantitative real-time PCR. Data are means $\pm S D$ from three

2 independent biological replicates. The asterisks indicate statistically significant

3 differences between the wild-type and WRKY45-kd leaves by the Student's t-test $(\mathrm{P}<$

4 0.05). e, Transactivation assay using a dual-luciferase system. The reporter construct

5 contained four W-box sequences upstream of the Firefly luciferase (F-Luc) coding

6 sequence. The Myc-WRKY45 construct contained a Myc-tagged full length WRKY45-

7 coding sequence downstream of the maize ubiquitin promoter (pUbi). The PBI1 construct

8 contained the PB/1-coding region downstream of the cauliflower mosaic virus $35 \mathrm{~S}$

9 promoter. The reference construct contained the Renilla luciferase (R-Luc) coding sequence downstream of the maize ubiquitin promoter. Luciferase activities were normalized against the reference R-Luc activity. Values are mean $\pm S$.E. Different letters above the data points indicate significant differences $(p<0.01$, Welch's test).

Fig. 5. PBI1 negatively regulates disease resistance through WRKY45.

a, PBI1 protein levels in leaves of the pbi1-knockout (ko) mutants pbi1-1 and pbi1-2 were analyzed by immunoblotting with $\mathbf{\alpha}-\mathrm{PBI}$. b, Phenotypes of the pbi1-ko mutants. c, WRKY45 protein levels in leaves of the pbi1-ko mutants, analyzed by immunoblotting with a-WRKY45. d, WRKY45 transcripts level in leaves of the pbi1 mutants, analyzed by quantitative real-time PCR. e, Rice leaves were infected with XooT7174 using a crimping method. The photograph of disease lesions was taken at $14 \mathrm{dpi}$. Scale bar $=1 \mathrm{~cm}$. $\mathbf{f}$, Mean lengths of disease lesions at 25 dpi. g, The bacterial populations of Xoo T7174 were analyzed by quantitative real-time PCR. The data indicate the DNA levels of the $X$. oryzae XopA gene relative to that of the rice ubiquitin gene. $\mathbf{h}$, Expression of WRKY62

24 in pbi1 suspension-cultured cells treated with $2 \mu \mathrm{g} / \mathrm{ml}$ (GluNAc) $)_{7}$, analyzed by 
1 quantitative real-time PCR. Error bars in $(\mathbf{d}),(\mathbf{f}),(\mathbf{g})$, and $(\mathrm{H})$ indicate $\pm S D$. Asterisks

2 indicate significant differences between the WT and the pbi1 mutants $(P<0.01)$.

3

Fig. 6. MAPKs regulate PBI1 degradation.

a, Chitin-induced MAPK activation in two mapkkk11/mapkkk18 mutants. Total proteins were prepared from rice suspension-cultured cells after treatment with $2 \mu \mathrm{g} / \mathrm{ml}$ (GluNAc) 7 and subjected to immunoblots with a-pMAPK. b, Chitin-induced PBI1 degradation was inhibited in the mapkkk11/mapkkk18 mutants. Total proteins were prepared as for (a) and probed with a-PBI1. c, The interactions between PBI1 and full length WRKY45 or WRKY45 fragments were analyzed using split NanoLuc assays. Constructs were made to produce PBI1 fused to $\mathrm{LgBiT}$ and the WRKY45 fragments fused to SmBiT. Fluc was used as an internal control. Rice protoplasts were transfected with the constructs and the interactions were indicated by the Nluc to Fluc ratios. Different letters above the data points indicate significant differences ( $p<0.01$, Welch's $t$ test). $\mathbf{d}$, Phosphorylation of WRKY45 inhibits the interaction between PBI1 and WRKY45. Split NanoLuc assays were carried out by transient expression of PBI1-LgBiT and WRKY45-SmBiT or WRKY $45^{\mathrm{DD}}-\mathrm{SmBiT}$ with or without $\mathrm{MKK} 4^{\mathrm{DD}}$ in rice protoplasts. Values are means $\pm \mathrm{S}$.E. Different letters above the data points indicate significant differences $(p<0.01$, Welch's t test). e, The expression levels of WRKY45 and WRKY62 in mapkkk11/mapkkk18 suspension-cultured cells treated with $2 \mu \mathrm{g} / \mathrm{ml}$ (GluNAc) $)_{7}$ were analyzed using quantitative real-time PCR. Data are means $\pm S D$ from three independent biological replicates, where each biological replicate consisted of two technical replicates. The asterisks indicate statistically significant differences from the WT controls by Student's ttest $(\mathrm{P}<0.05)$. 
Fig. 7. PUB44 is phosphorylated upon chitin perception.

a, A mobility shift of PUB44 was detected by immunoblotting with a-PUB44 using total proteins prepared from WT rice suspension-cultured cells after treatment with $2 \mu \mathrm{g} / \mathrm{ml}$

$6 \quad$ (GluNAc) 7 (upper panel). The arrow indicates the shifted PUB44 band. The mobility shift

7 did not occur in Oscerk1 mutant cells (lower panel). b. The mobility shift of PUB44 was

8 reversed by treatment with $\lambda$ protein phosphatase, indicating that the shift was due to

9 phosphorylation of PUB44. The arrow indicates the shifted PUB44 band. c, PUB44 phosphorylation was delayed and reduced in the mapkkk11/18 mutant (lower panel) when compared with WT cells (upper panel). d, OsCERK1 transcript levels in WT and mapkkk11/18 mutant cells, measured by quantitative real-time PCR. Data are means $\pm S D$ from three independent biological replicates, where each biological replicate consisted of two technical replicates. The asterisks indicate statistically significant differences from the WT controls by Student's t-test $(P<0.05)$. 


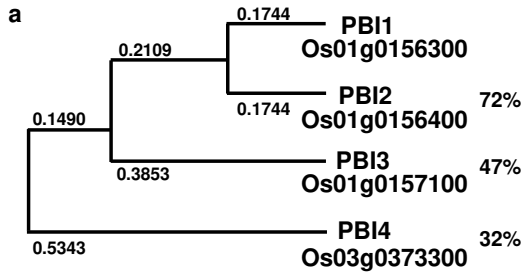

b

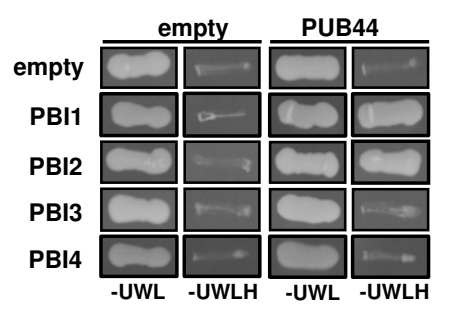

c

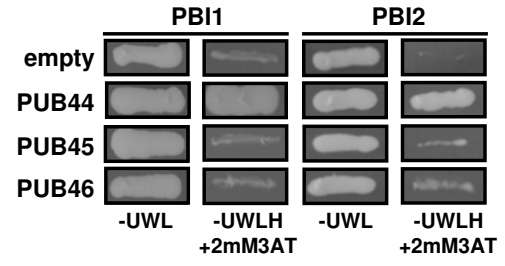

d
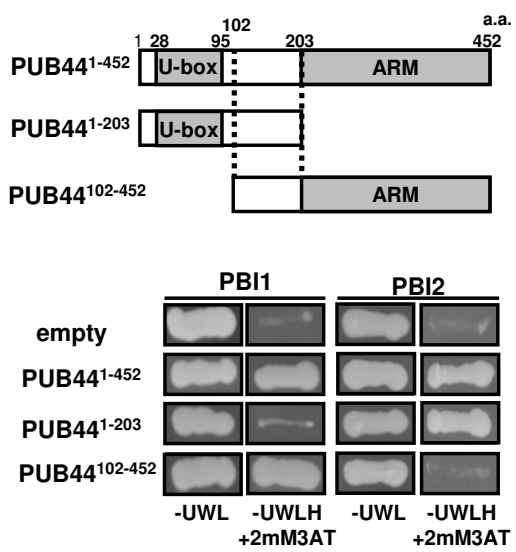

Fig. 1. Proteins containing the DUF1110 domain form a small protein family in rice.

a, Phylogenetic tree of the rice DUF1110 domain-containing proteins. Full-length protein sequences were used in the alignment. The neighbor-joining phylogenetic tree was created using ClustalW on the DNA Data Bank of Japan website (http://www.ddbj.nig.ac.jp/). The tree was generated from the modified alignment using Treeview $X$ software. The percentages indicate amino acid identity with PBI1. b, Interactions between PBI family members and PUB44 in yeast two-hybrid experiments. Growth of yeast colonies on -ULWH plates (lacking uracil, leucine, tryptophan, and histidine) indicates a positive interaction. c, Interactions of PBI1 and PBI2 with PUB44, PUB45, and PUB46 in yeast two-hybrid experiments. Growth of yeast colonies on -ULWH plates with $2 \mathrm{mM}$ 3-aminotriazole (3-AT) indicates a positive interaction. d, (Upper panel) Schematic diagram of PUB44 constructs. (Bottom panel) Interactions of PBI1 and PBI2 with each domain of PUB44 in yeast two-hybrid experiments. Positive interactions are as for (c). 

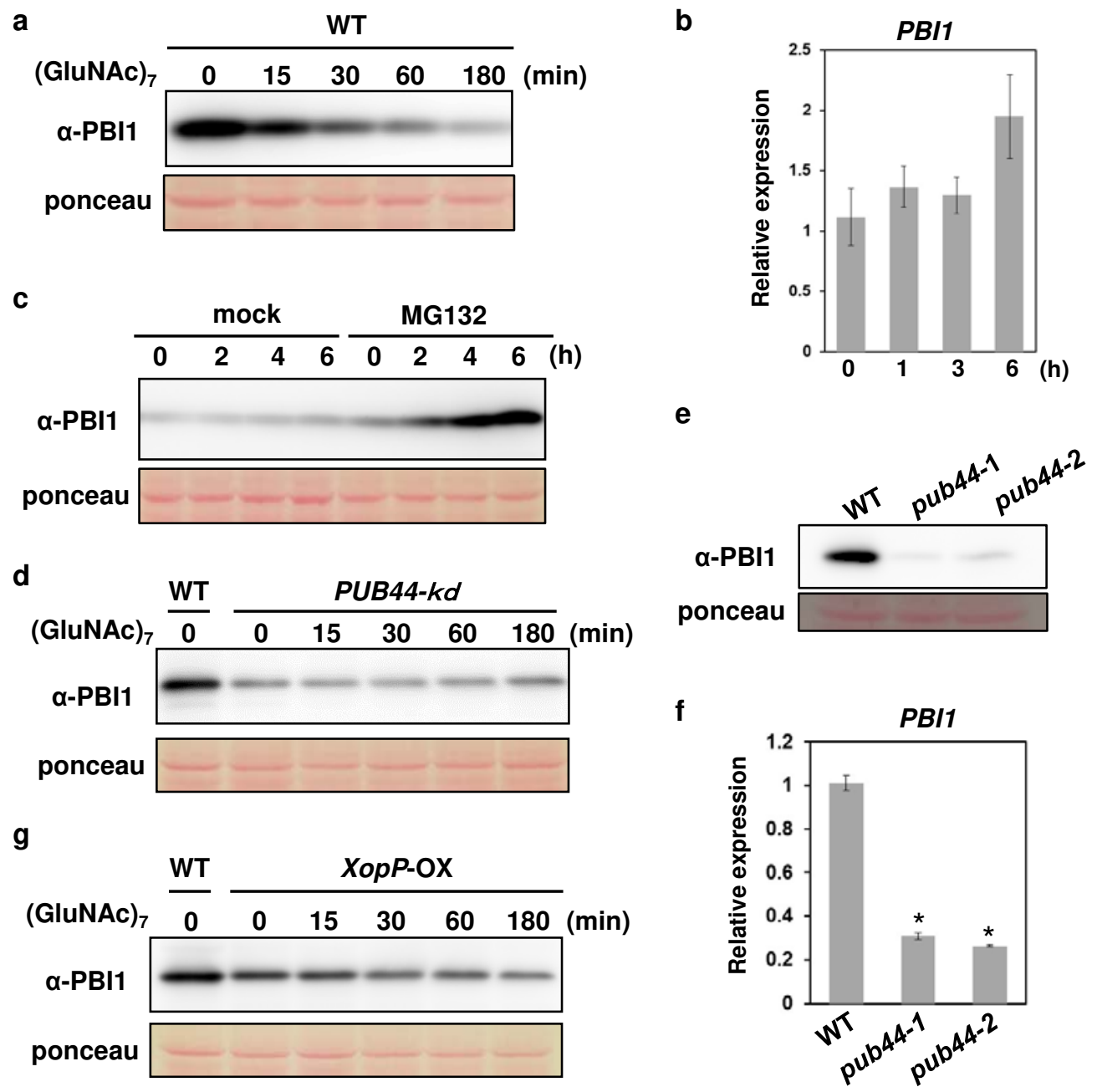

\section{Fig 2. Chitin-induced degradation of PBI1}

a, Total proteins were prepared from wildtype (WT) rice suspension-cultured cells after treatment with $2 \mu \mathrm{g}$ $\left(\right.$ GluNAc) ${ }_{7}$ and subjected to immunoblots with a-PBI1. b, PB/1 transcript levels in rice cells treated with $2 \mu \mathrm{g}$ $(\text { GluNAc })_{7}$ were analyzed using quantitative real-time PCR. Data are means \pm SD from three independent biological replicates, where each biological replicate consisted of two technical replicates. c,PBI1 protein levels in rice cells after treatment with the proteasome inhibitor MG132 $(30 \mu \mathrm{M})$ or dimethylsulphoxide (DMSO; mock), determined by immunoblot analysis with a-PBI1. d, PBI1 protein levels in PUB44 RNAi cells after treatment with $2 \mu \mathrm{g}(\mathrm{GluNAc})_{7}$, determined by immunoblot analysis with $\alpha$-PBI1. e, PBI1 protein levels in the PUB44 knockout mutants, determined by immunoblot analysis with $\alpha-P B \mid 1$. f, PB/1 transcript levels in the PUB44 knockout mutants, analyzed using quantitative real-time PCR. Data are means $\pm S D$ from three independent biological replicates, where each biological replicate consisted of two technical replicates. The asterisks indicate statistically significant differences from the WT controls by Student's t-test $(\mathrm{P}<0.05)$. g, PBI1 protein levels in XopP-ox cells after treatment with $2 \mu \mathrm{g}(\mathrm{GluNAc})_{7}$, determined by immunoblot analysis with $\alpha-\mathrm{PB} 11$. 
a

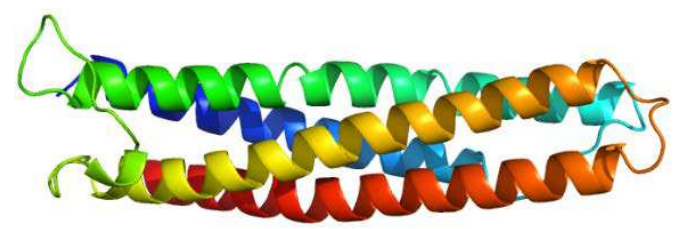

b

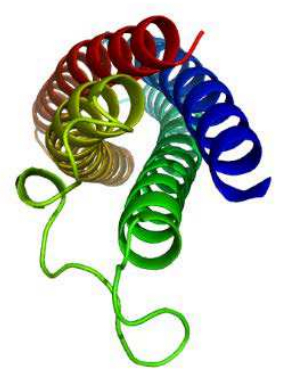

C
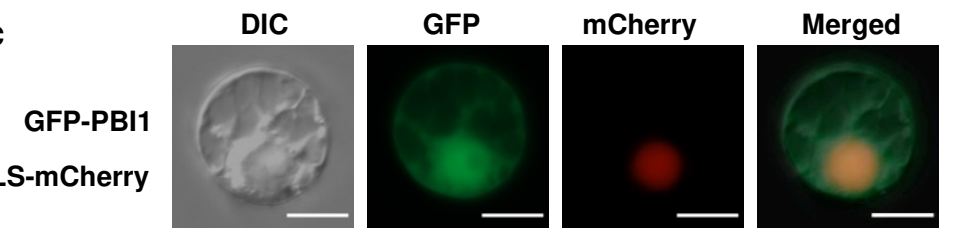

PBI1-GFP

NLS-mCherry
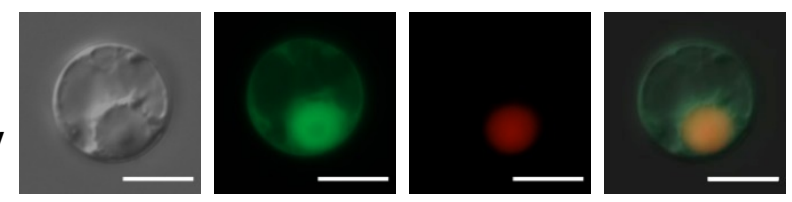

Fig 3. PBI1, with a four-helix bundle structure, localizes mainly to the nucleus.

a, Side view of PBI1, which forms a four-helix bundle. Coloring is from blue at the N-terminus to red at the C-terminus. b, End view, with $\mathrm{N}$ - and C-termini at the front. c, Detection of GFP-PBI1 and PBI1-GFP after transient expression in rice protoplasts. mCherry with a nuclear localization signal was used as a nuclear localization marker. Scale bar $=10 \mu \mathrm{m}$. 
a

PBI1-Vc WRKY45-Vn
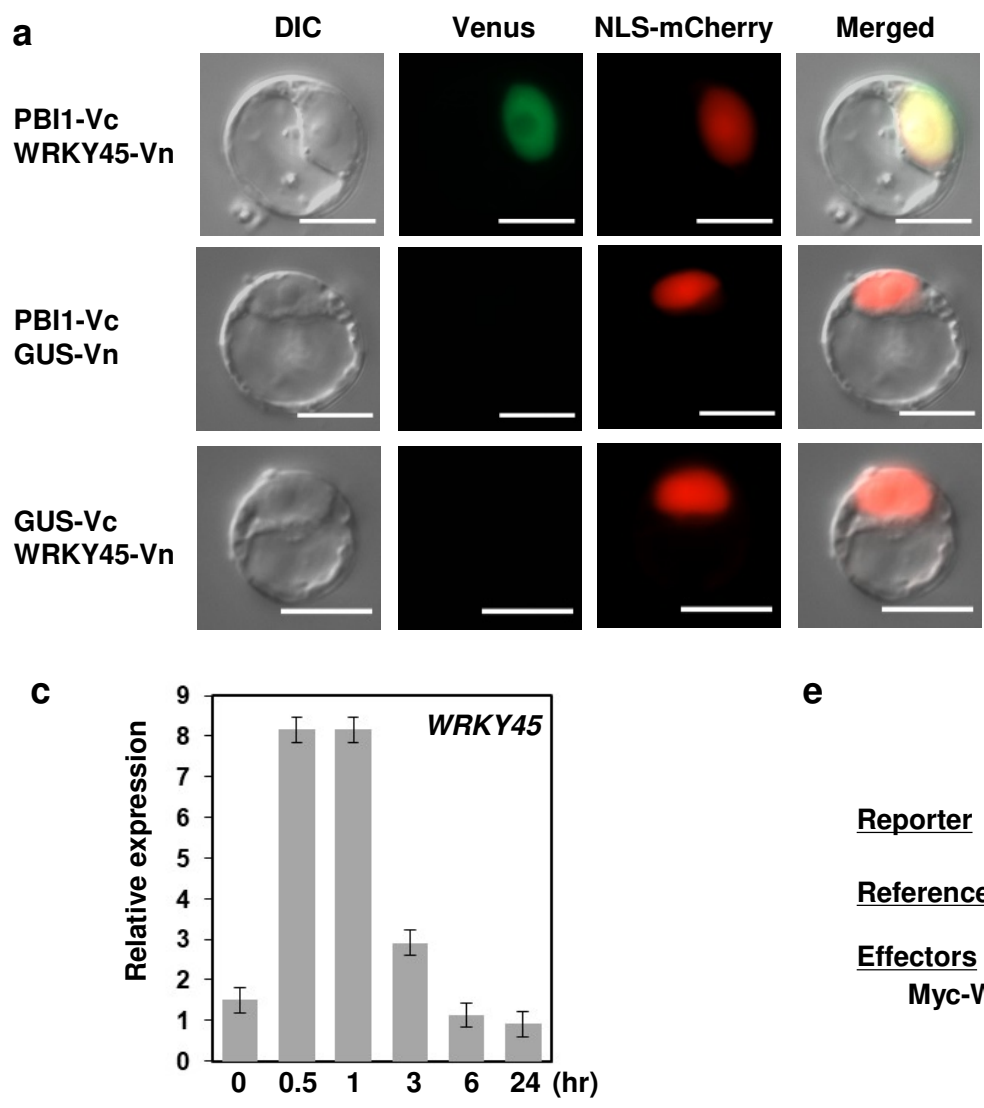

d

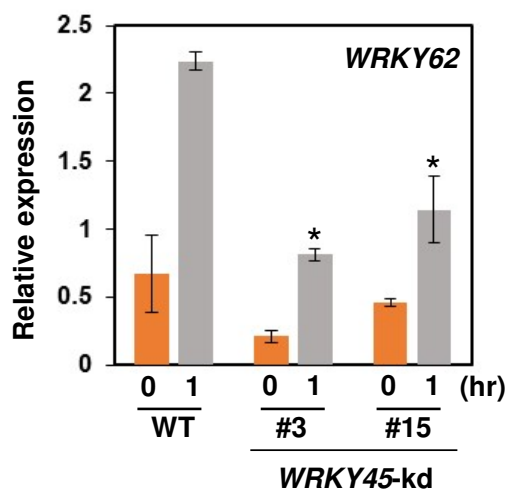

C b
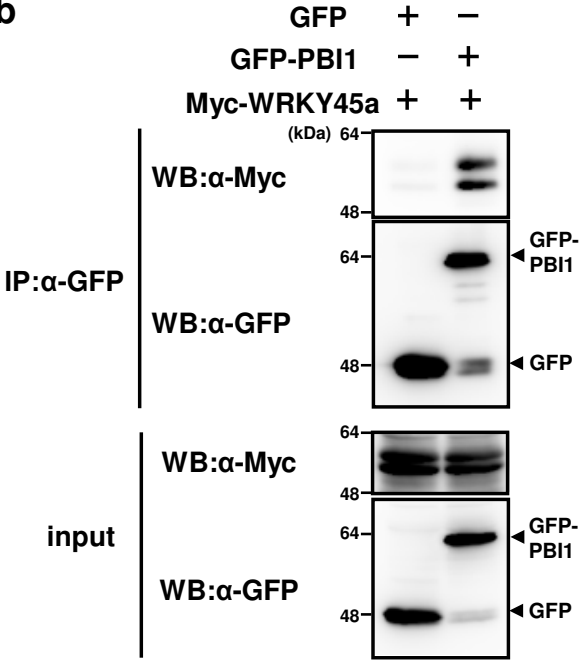

Fig 4. PBI1 interacts with and inhibits WRKY45

a, Bimolecular fluorescence complementation (BiFC) analysis was used to visualize the interaction between PBI1-Vc and WRKY45$\mathrm{Vn}$ in rice protoplasts. mCherry with a nuclear localization signal was used as a nuclear localization marker. The $\beta$-glucuronidase (GUS) protein was used as a negative control. Scale bar $=10 \mu \mathrm{m}$. b. Rice protoplasts were co-transfected with GFP-PBI1 and Myctagged WRKY45 and subjected to a co-immunoprecipitation assay. Proteins were precipitated using an antibody against GFP ( $\alpha-$ GFP), and the input proteins and precipitated proteins were probed with a-Myc and $\alpha$-GFP. c, WRKY45 transcript levels in rice suspension-cultured cells treated with $2 \mu \mathrm{g}$ (GluNAc) $)_{7}$ were analyzed using quantitative real-time PCR. d, Expression levels of WRKY62 in wild type and WRKY45-knockdown (kd) leaves after treatment with $2 \mu \mathrm{g}(\mathrm{GluNAc})_{7}$, analyzed using quantitative realtime PCR. Data are means $\pm S D$ from three independent biological replicates. The asterisks indicate statistically significant differences between the wild-type and WRKY45-kd leaves by the Student's t-test $(P<0.05)$. e, Transactivation assay using a dualluciferase system. The reporter construct contained four W-box sequences upstream of the Firefly luciferase (F-Luc) coding sequence. The Myc-WRKY45 construct contained a Myc-tagged full length WRKY45-coding sequence downstream of the maize ubiquitin promoter (pUbi). The PBI1 construct contained the PB/1-coding region downstream of the cauliflower mosaic virus $35 \mathrm{~S}$ promoter. The reference construct contained the Renilla luciferase (R-Luc) coding sequence downstream of the maize ubiquitin promoter. Luciferase activities were normalized against the reference R-Luc activity. Values are mean $\pm S$.E. Different letters above the data points indicate significant differences $(p<0.01$, Welch's $t$ test). 

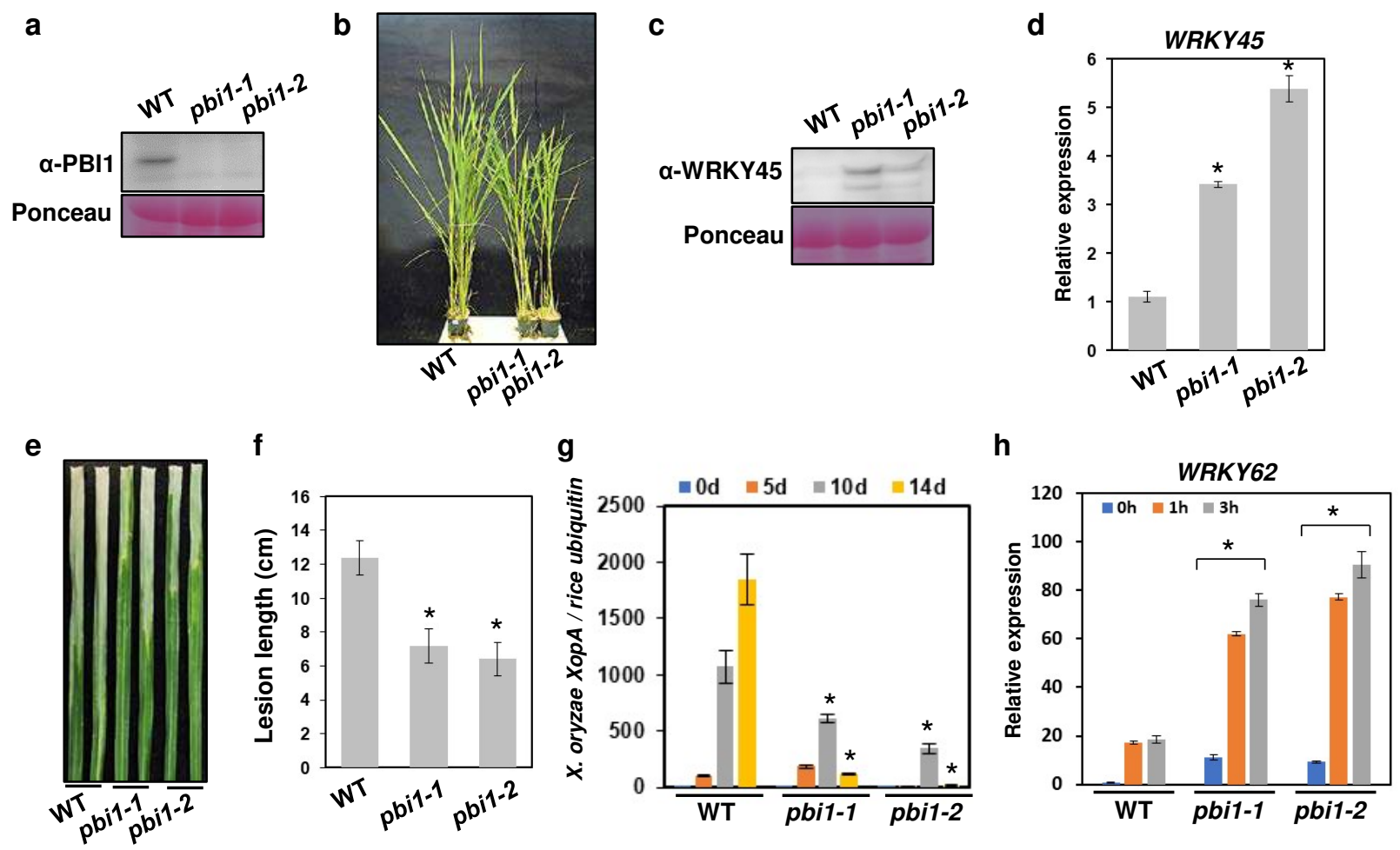

Fig 5. PBI1 negatively regulates disease resistance through WRKY45.

a, PBI1 protein levels in leaves of the pbi1-knockout ( $k$ o) mutants pbi1-1 and pbi1-2 were analyzed by immunoblotting with a-PBI. b. Phenotypes of the pbi1-ko mutants. c, WRKY45 protein levels in leaves of the pbi1-ko mutants, analyzed by immunoblotting with a-WRKY45. d, WRKY45 transcripts level in leaves of the pbi1 mutants, analyzed by quantitative real-time PCR. e, Rice leaves were infected with Xoo T7174 using a crimping method. The photograph of disease lesions was taken at $14 \mathrm{dpi}$. Scale bar $=1 \mathrm{~cm}$. $\mathbf{f}$, Mean lengths of disease lesions at $25 \mathrm{dpi}$. $\mathbf{g}$. The bacterial populations of Xoo T7174 were analyzed by quantitative real-time PCR. The data indicate the DNA levels of the $X$. oryzae XopA gene relative to that of the rice ubiquitin gene. $\mathbf{h}$, Expression of WRKY62 in pbi1 suspension-cultured cells treated with $2 \mu \mathrm{g}$ $(\text { GluNAc) })_{7}$, analyzed by quantitative real-time PCR. Error bars in (d), (f), (g), and (h) indicate \pm SD. Asterisks indicate significant differences between the WT and the pbi1 mutants $(P<0.01)$. 
a
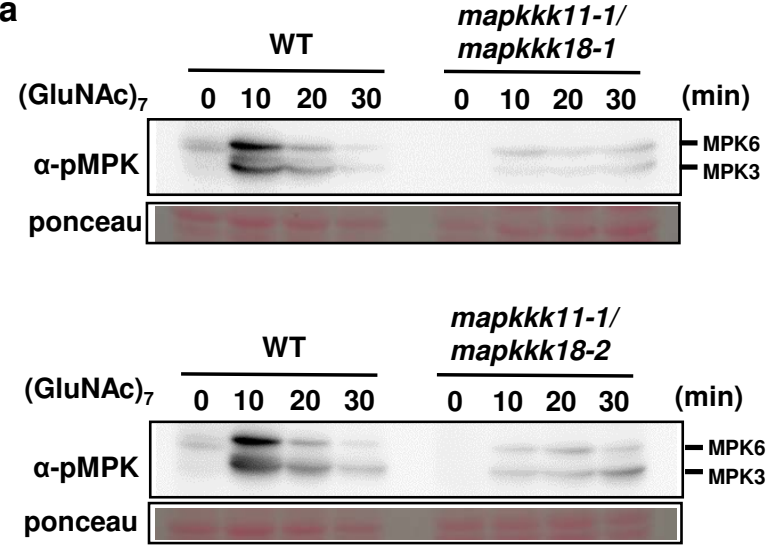

C

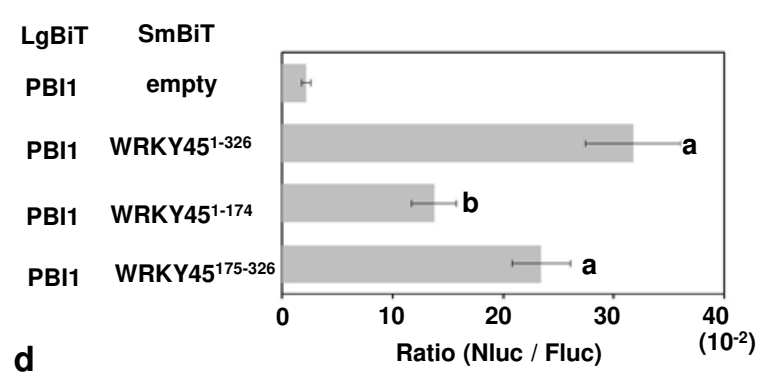

LgBiT SmBiT Effector

PBI1 empty

PBI1 WRKY45

PBI1 WRKY45 ${ }^{D D}$

PBI1 WRKY45

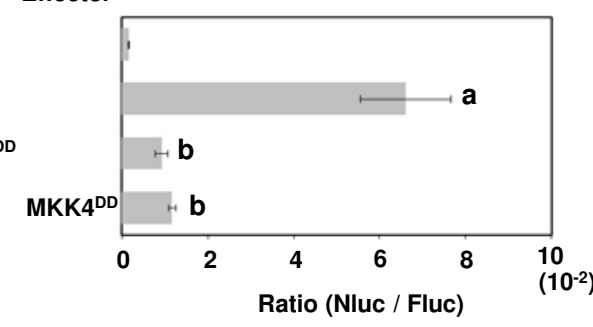

b
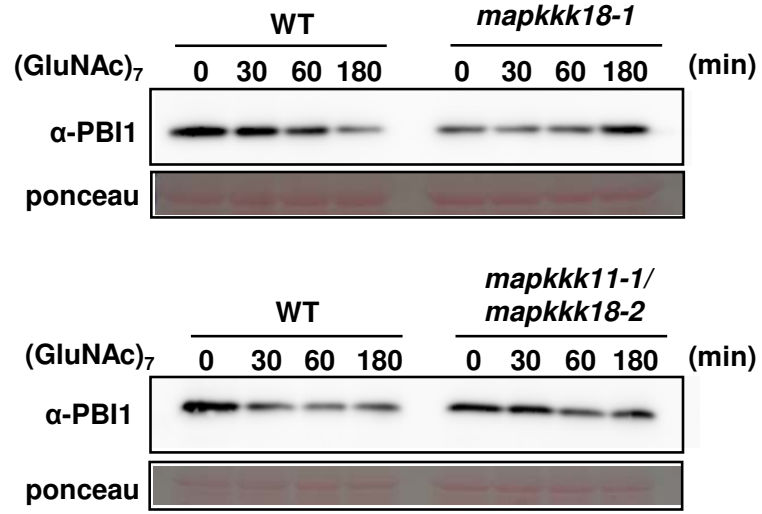

e
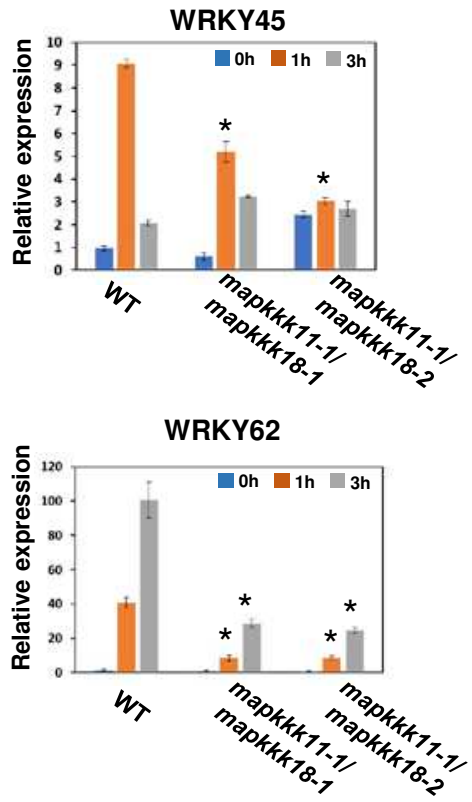

Fig 6. MAPKs regulate PBI1 degradation.

a, Chitin-induced MAPK activation in two mapkkk11/mapkkk18 mutants. Total proteins were prepared from rice suspension-cultured cells after treatment with $2 \mu \mathrm{g}$ (GluNAc) $)_{7}$ and subjected to immunoblots with $\alpha$-pMAPK. b, Chitin-induced PBI1 degradation was inhibited in the mapkkk11/mapkkk18 mutants. Total proteins were prepared as for (a) and probed with $\alpha$-PBI1. c, The interactions between PBI1 and full length WRKY45 or WRKY45 fragments were analyzed using split NanoLuc assays. Constructs were made to produce PBI1 fused to LgBiT and the WRKY45 fragments fused to SmBiT. Fluc was used as an internal control. Rice protoplasts were transfected with the constructs and the interactions were indicated by the Nluc to Fluc ratios. Different letters above the data points indicate significant differences ( $p<0.01$, Welch's $t$ test). d, Phosphorylation of WRKY45 inhibits the interaction between PBI1 and WRKY45. Split NanoLuc assays were carried out by transient expression of PBI1$\mathrm{LgBiT}$ and WRKY45-SmBiT or WRKY45 ${ }^{\mathrm{DD}}-\mathrm{SmBiT}$ with or without MKK4 ${ }^{\mathrm{DD}}$ in rice protoplasts. Values are means \pm S.E. Different letters above the data points indicate significant differences ( $p<0.01$, Welch's $t$ test). e, The expression levels of WRKY45 and WRKY62 in mapkkk11/mapkkk18 suspension-cultured cells treated with $2 \mu \mathrm{g}$ $(\text { GluNAc })_{7}$ were analyzed using quantitative real-time PCR. Data are means $\pm S D$ from three independent biological replicates, where each biological replicate consisted of two technical replicates. The asterisks indicate statistically significant differences from the WT controls by Student's t-test $(P<0.05)$. 

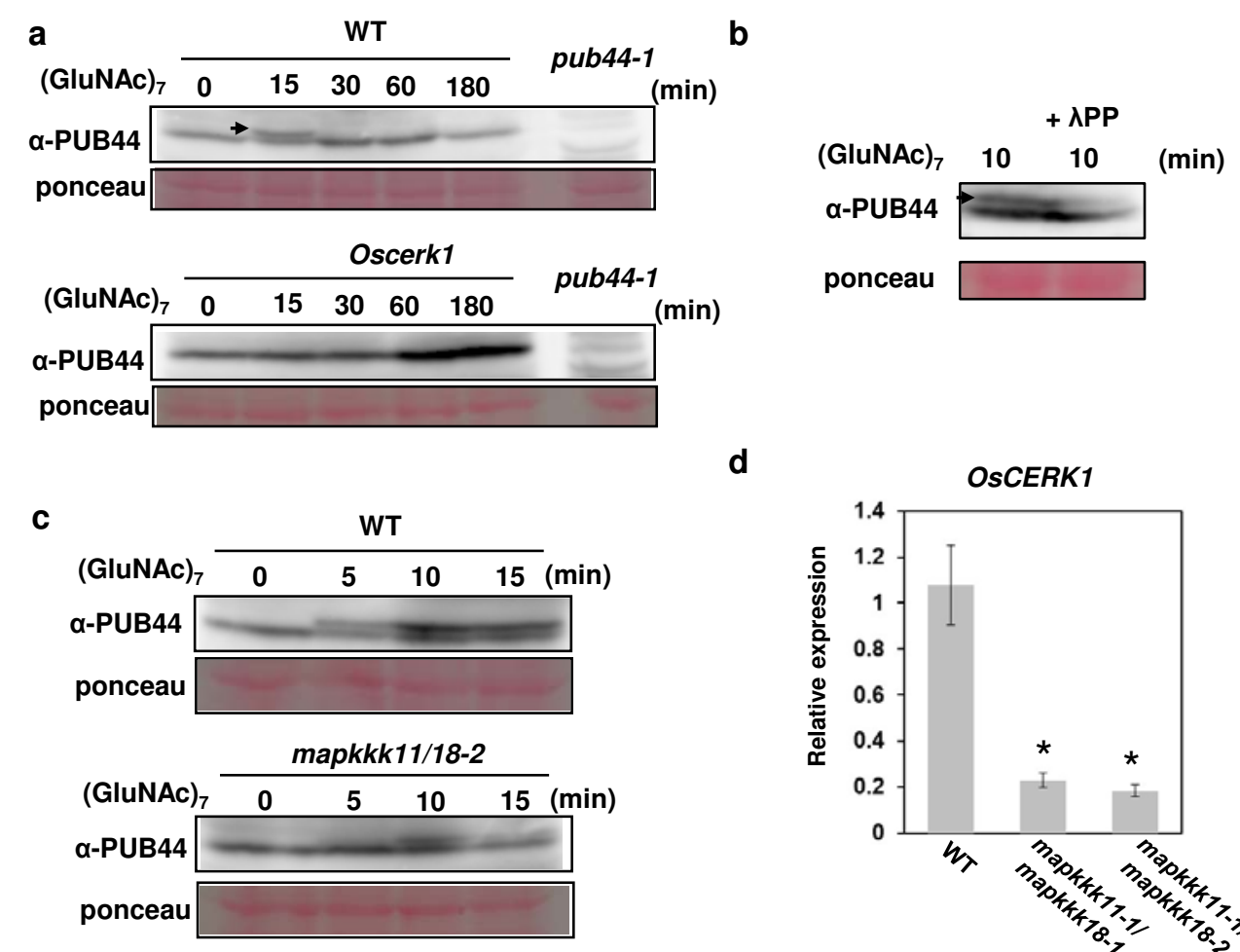

d

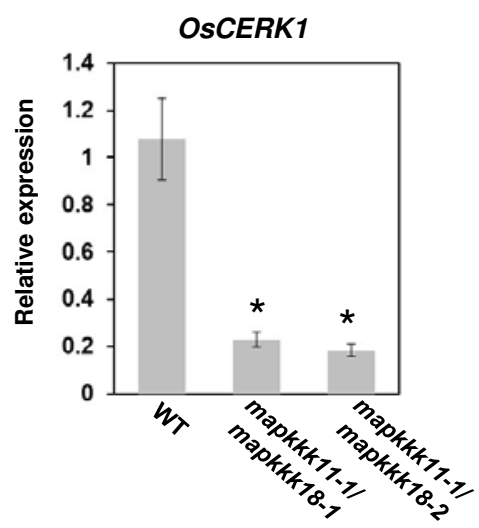

Fig 7. PUB44 is phosphorylated upon chitin perception.

a, A mobility shift of PUB44 was detected by immunoblotting with a-PUB44 using total proteins prepared from

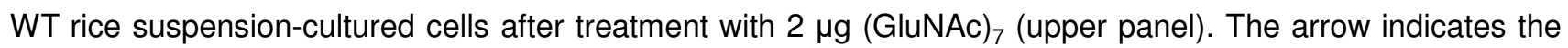
shifted PUB44 band. The mobility shift did not occur in Oscerk1 mutant cells (lower panel). b. The mobility shift of PUB44 was reversed by treatment with $\lambda$ protein phosphatase, indicating that the shift was due to phosphorylation of PUB44. The arrow indicates the shifted PUB44 band. c, PUB44 phosphorylation was delayed and reduced in the mapkkk11/18 mutant (lower panel) when compared with WT cells (upper panel). d, OSCERK1 transcript levels in WT and mapkkk11/18 mutant cells, measured by quantitative real-time PCR. Data are means \pm SD from three independent biological replicates, where each biological replicate consisted of two technical replicates. The asterisks indicate statistically significant differences from the WT controls by Student's t-test $(P<0.05)$. 


\section{Figures}

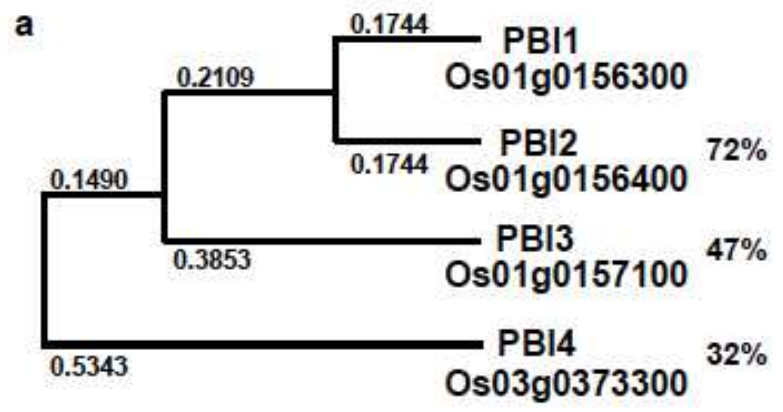

b

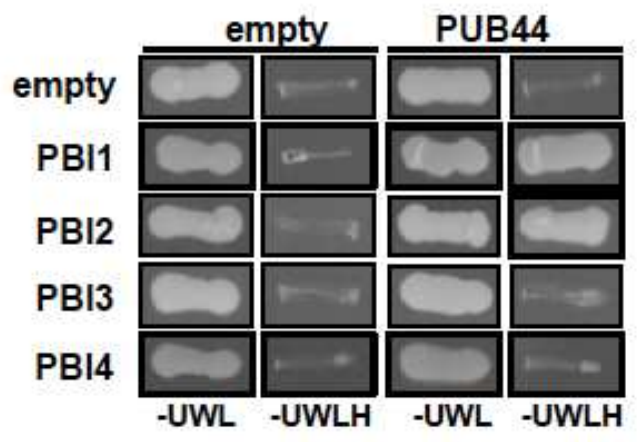

c

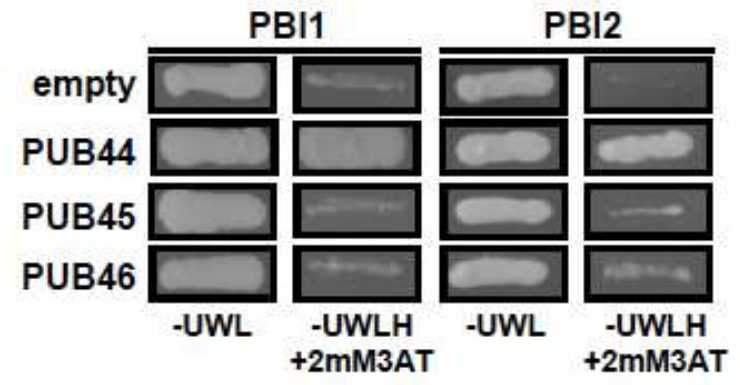

d
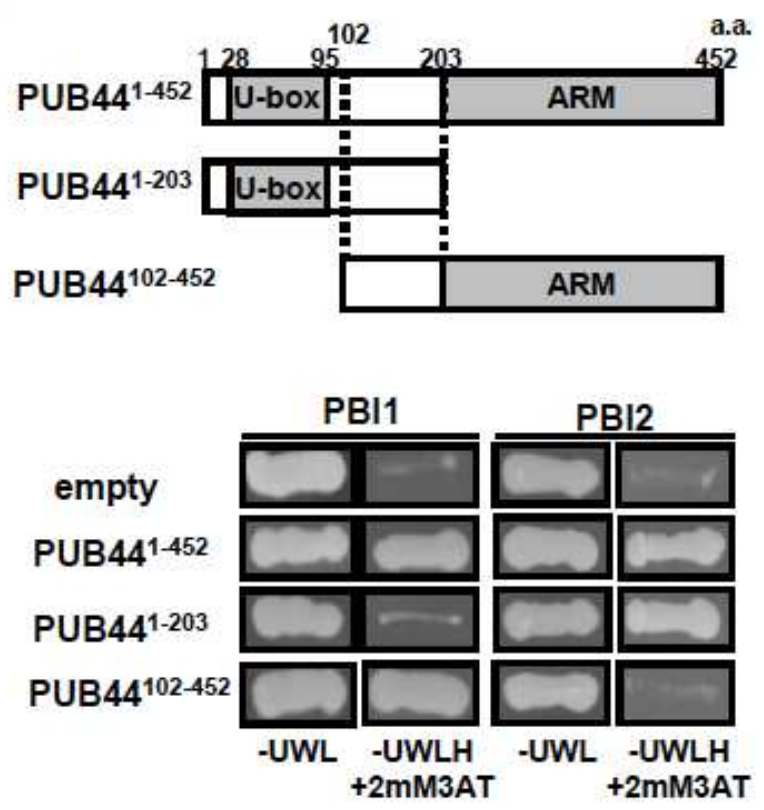

\section{Figure 1}

Proteins containing the DUF1110 domain form a small protein family in rice. a, Phylogenetic tree of the rice DUF1110 domain-containing proteins. Full-length protein sequences were used in the alignment. The neighbor-joining phylogenetic tree was created using ClustalW on the DNA Data Bank of Japan website (http://www.ddbj.nig.ac.jp/). The tree was generated from the modified alignment using Treeview $X$ software. The percentages indicate amino acid identity with PBI1. b, Interactions between PBI family members and PUB44 in yeast two-hybrid experiments. Growth of yeast colonies on -ULWH plates (lacking uracil, leucine, tryptophan, and histidine) indicates a positive interaction. c, Interactions of PBI1 and PBI2 with PUB44, PUB45, and PUB46 in yeast two-hybrid experiments. Growth of yeast colonies on ULWH plates with 2 mM 3-aminotriazole (3-AT) indicates a positive interaction. d, (Upper panel) Schematic diagram of PUB44 constructs. (Bottom panel) Interactions of PBI1 and PBI2 with each domain of PUB44 in yeast two-hybrid experiments. Positive interactions are as for (c). 
a

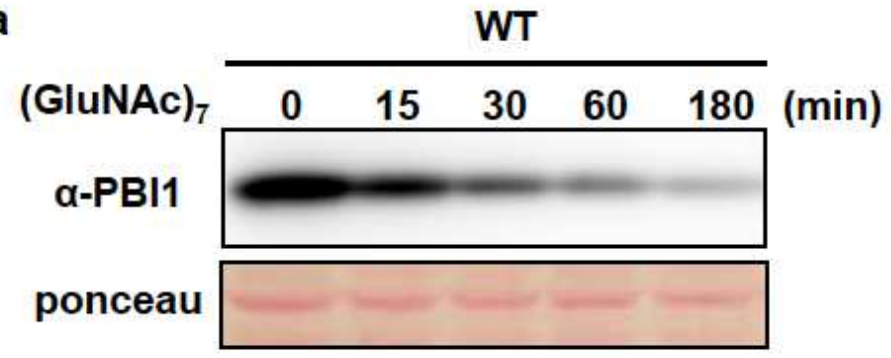

c
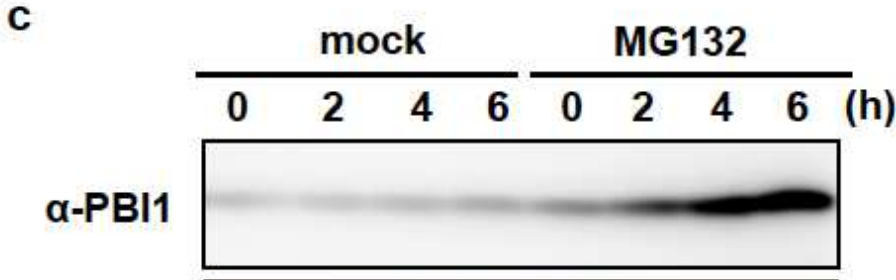

ponceau

d

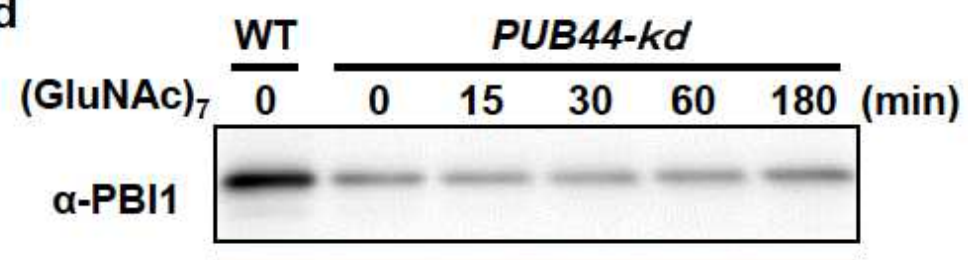

ponceau

g

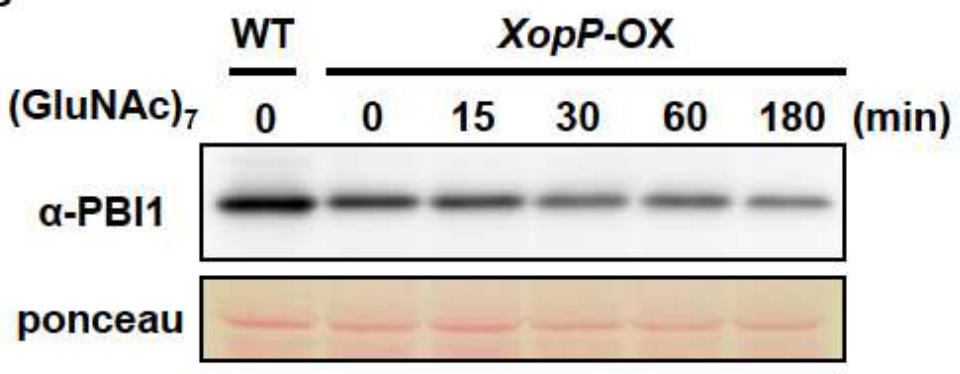

b

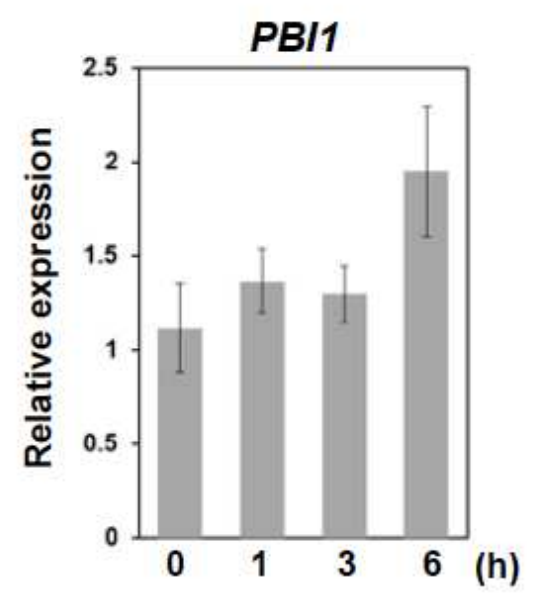

e

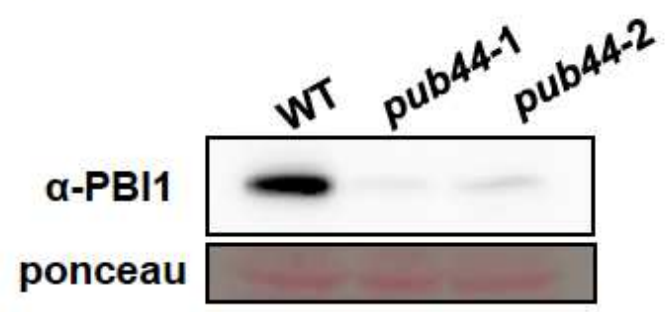

f

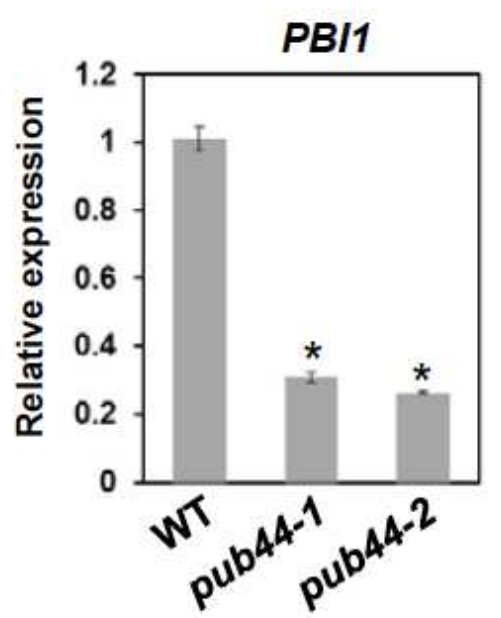

Figure 2

Chitin-induced degradation of PBI1 a, Total proteins were prepared from wildtype (WT) rice suspensioncultured cells after treatment with $2 \mu \mathrm{g}$ (GluNAc) 7 and subjected to immunoblots with a-PBI1. b, PBI1 transcript levels in rice cells treated with $2 \mu \mathrm{g}$ (GluNAc) 7 were analyzed using quantitative real-time PCR. Data are means \pm SD from three independent biological replicates, where each biological replicate consisted of two technical replicates. c,PBI1 protein levels in rice cells after treatment with the proteasome inhibitor MG132 (30 $\mu \mathrm{M})$ or dimethylsulphoxide (DMSO; mock), determined by immunoblot analysis with a-PBI1. d, PBI1 protein levels in PUB44 RNAi cells after treatment with $2 \mu \mathrm{g}$ (GluNAc)7, determined by immunoblot analysis with a-PBI1. e, PBI1 protein levels in the PUB44 knockout mutants, 
determined by immunoblot analysis with a-PBI1. f, PBI1 transcript levels in the PUB44 knockout mutants, analyzed using quantitative real-time PCR. Data are means \pm SD from three independent biological replicates, where each biological replicate consisted of two technical replicates. The asterisks indicate statistically significant differences from the WT controls by Student's t-test $(P<0.05)$. g, PBI1 protein levels in XopP-ox cells after treatment with $2 \mu \mathrm{g}$ (GluNAc)7, determined by immunoblot analysis with aPBI1.

a

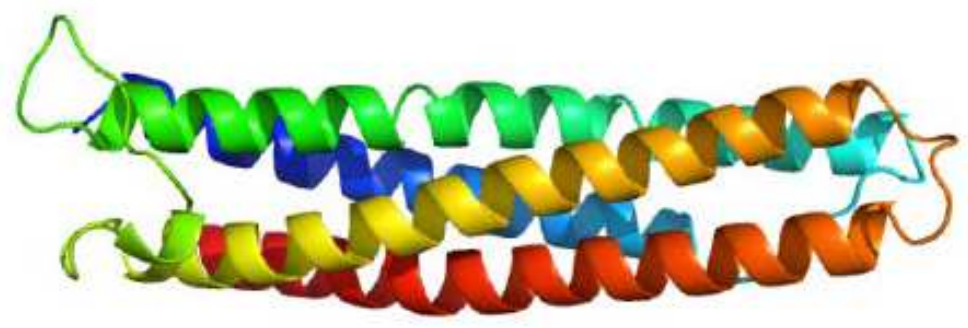

b

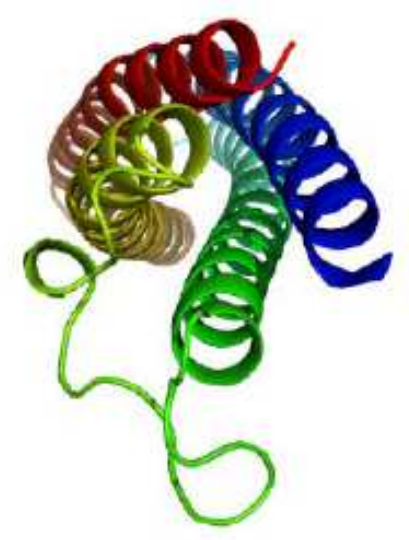

C

DIC
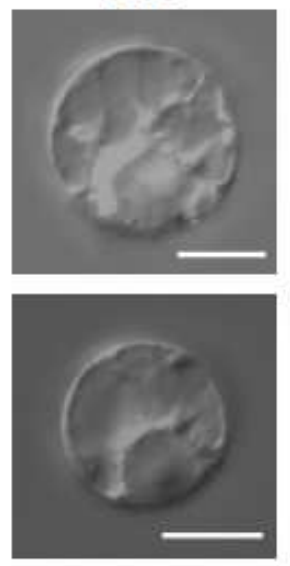

GFP
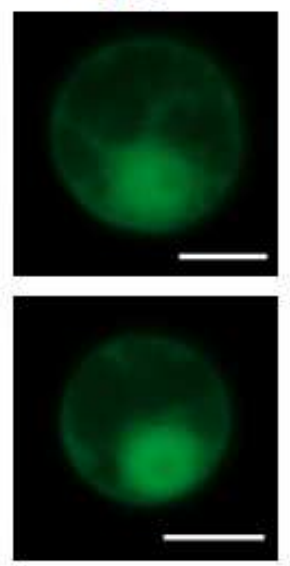

mCherry
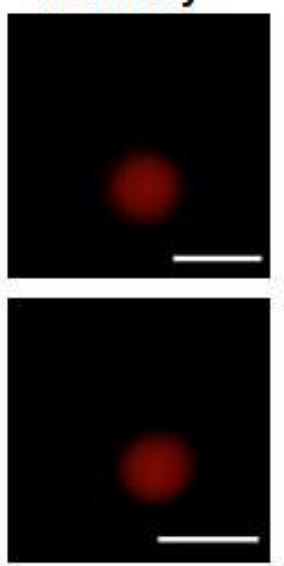

Merged
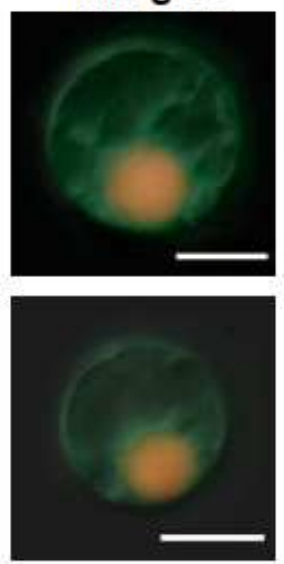

Figure 3 
PBI1, with a four-helix bundle structure, localizes mainly to the nucleus. a, Side view of PBI1, which forms a four-helix bundle. Coloring is from blue at the $\mathrm{N}$-terminus to red at the C-terminus. b, End view, with $\mathrm{N}$ and C-termini at the front. c, Detection of GFP-PBI1 and PBI1-GFP after transient expression in rice protoplasts. mCherry with a nuclear localization signal was used as a nuclear localization marker. Scale bar $=10 \mu \mathrm{m}$.

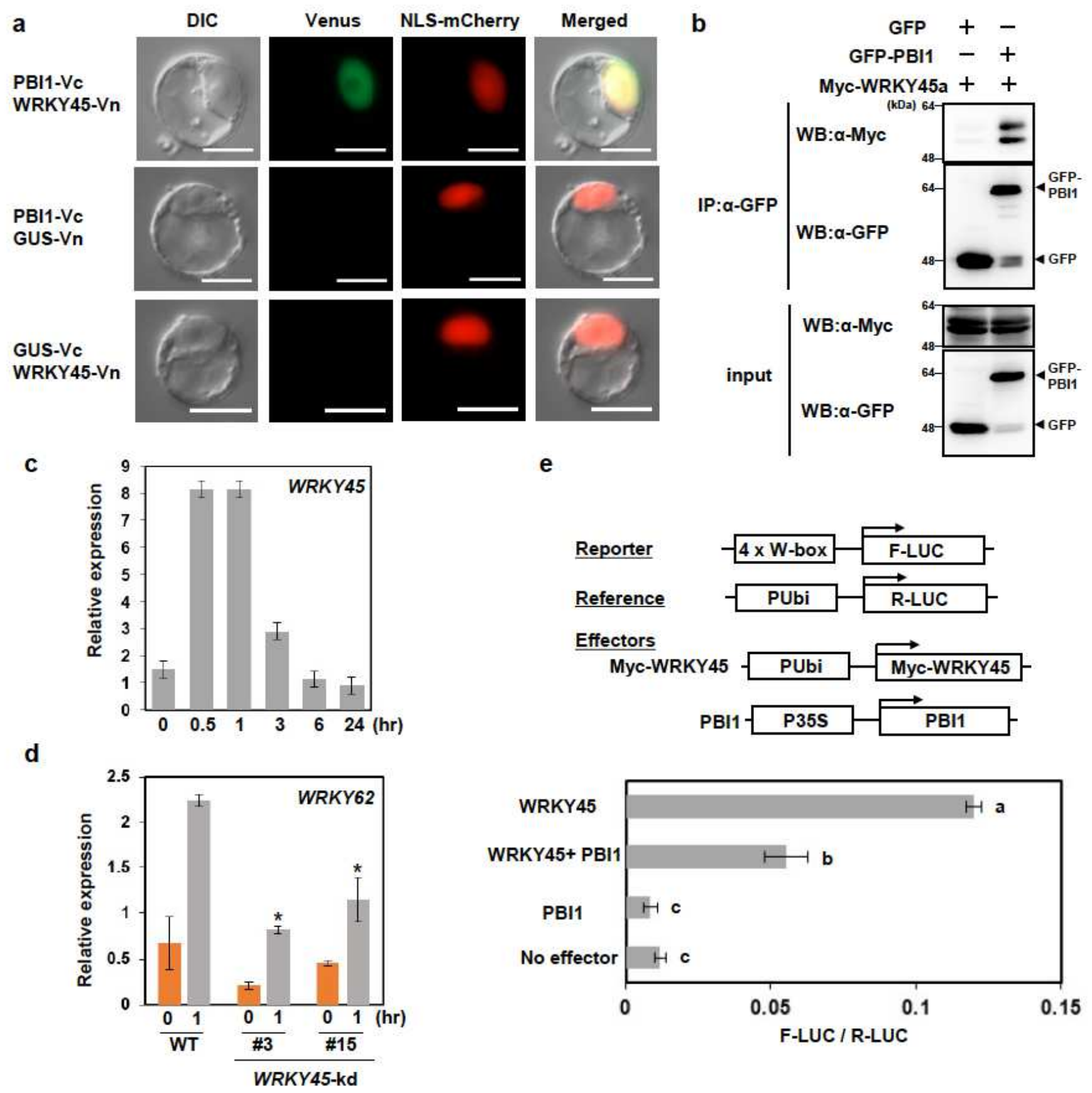

\section{Figure 4}

PBI1 interacts with and inhibits WRKY45 a, Bimolecular fluorescence complementation (BiFC) analysis was used to visualize the interaction between PBI1-Vc and WRKY45- $\mathrm{Vn}$ in rice protoplasts. mCherry with a nuclear localization signal was used as a nuclear localization marker. The $\beta$-glucuronidase (GUS) protein was used as a negative control. Scale bar $=10 \mu \mathrm{m}$. b, Rice protoplasts were co-transfected with 
GFP-PBI1 and Myc- tagged WRKY45 and subjected to a co-immunoprecipitation assay. Proteins were precipitated using an antibody against GFP (a-GFP), and the input proteins and precipitated proteins were probed with a-Myc and a-GFP. c, WRKY45 transcript levels in rice suspension-cultured cells treated with $2 \mu \mathrm{g}$ (GluNAc)7 were analyzed using quantitative real-time PCR. d, Expression levels of WRKY62 in wild type and WRKY45-knockdown (kd) leaves after treatment with $2 \mu \mathrm{g}$ (GluNAc)7, analyzed using quantitative real- time PCR. Data are means \pm SD from three independent biological replicates. The asterisks indicate statistically significant differences between the wild-type and WRKY45-kd leaves by the Student's t-test $(P<0.05)$. e, Transactivation assay using a dual- luciferase system. The reporter construct contained four W-box sequences upstream of the Firefly luciferase (F-Luc) coding sequence. The MycWRKY45 construct contained a Myc-tagged full length WRKY45-coding sequence downstream of the maize ubiquitin promoter (pUbi). The PBI1 construct contained the PBI1-coding region downstream of the cauliflower mosaic virus 35S promoter. The reference construct contained the Renilla luciferase (R-Luc) coding sequence downstream of the maize ubiquitin promoter. Luciferase activities were normalized against the reference R-Luc activity. Values are mean \pm S.E. Different letters above the data points indicate significant differences ( $p<0.01$, Welch's t test).
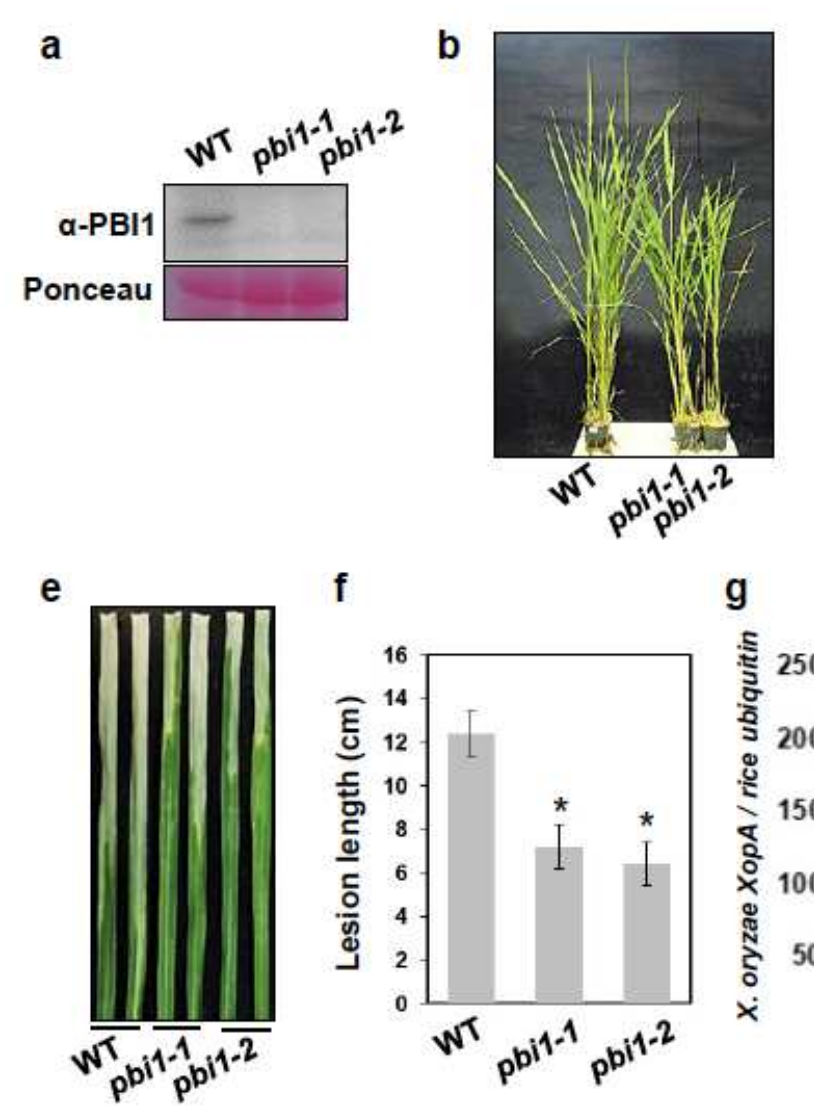

$f$

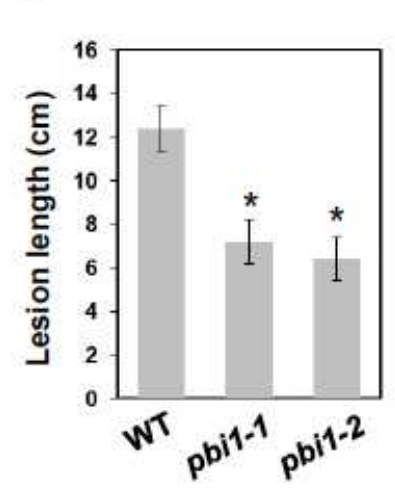

C

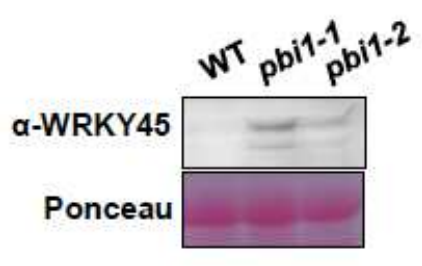

g

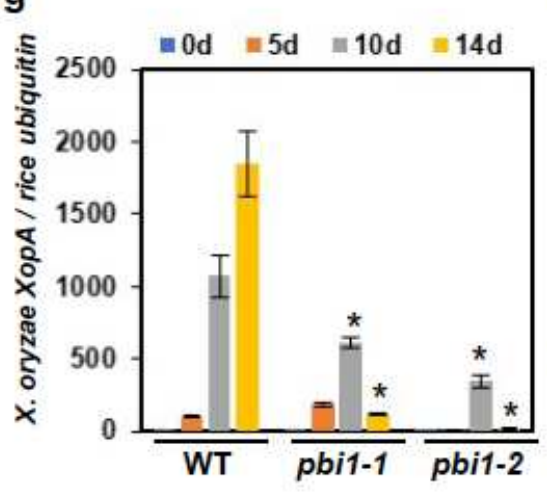

d

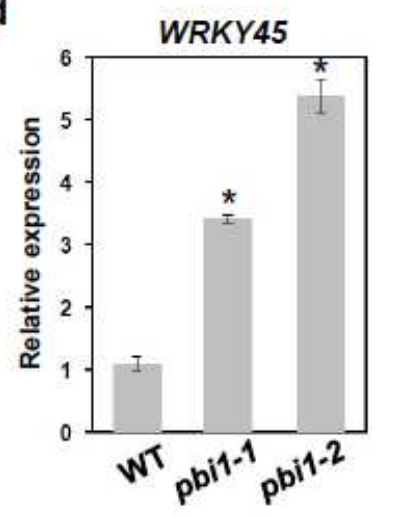

h

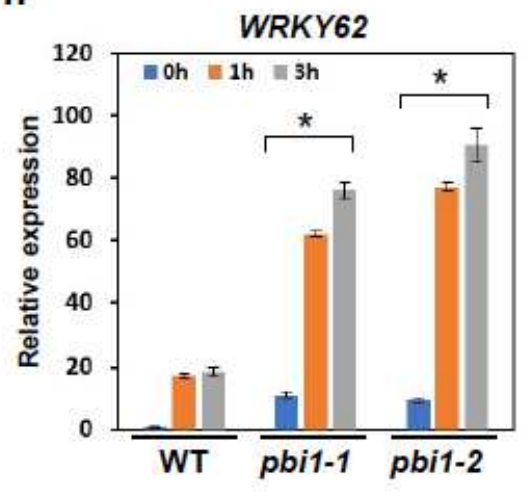

\section{Figure 5}

PBI1 negatively regulates disease resistance through WRKY45. a, PBI1 protein levels in leaves of the pbi1knockout (ko) mutants pbi1-1 and pbi1-2 were analyzed by immunoblotting with a-PBI. b, Phenotypes of 
the pbi1-ko mutants. c, WRKY45 protein levels in leaves of the pbi1-ko mutants, analyzed by immunoblotting with a-WRKY45. d, WRKY45 transcripts level in leaves of the pbi1 mutants, analyzed by quantitative real-time PCR. e, Rice leaves were infected with Xoo T7174 using a crimping method. The photograph of disease lesions was taken at $14 \mathrm{dpi}$. Scale bar $=1 \mathrm{~cm}$. f, Mean lengths of disease lesions at $25 \mathrm{dpi}$. g. The bacterial populations of Xoo T7174 were analyzed by quantitative real-time PCR. The data indicate the DNA levels of the X. oryzae XopA gene relative to that of the rice ubiquitin gene. $h$, Expression of WRKY62 in pbi1 suspension-cultured cells treated with $2 \mu \mathrm{g}$ (GluNAc)7, analyzed by quantitative real-time PCR. Error bars in (d), (f), (g), and (h) indicate $\pm S D$. Asterisks indicate significant differences between the WT and the pbi1 mutants $(P<0.01)$.

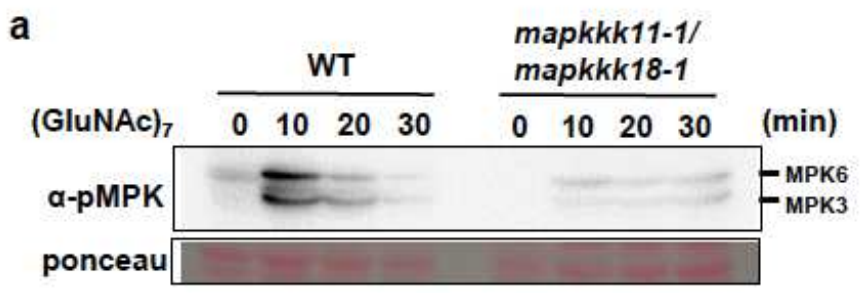

b
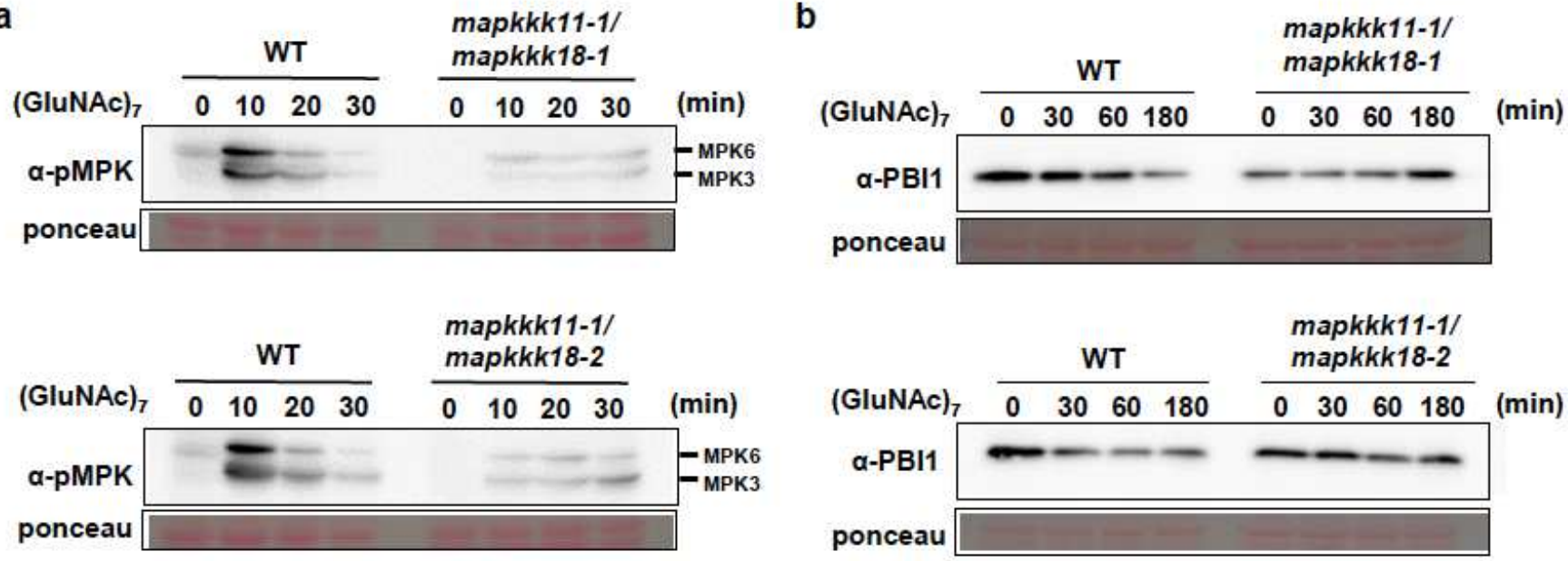

C

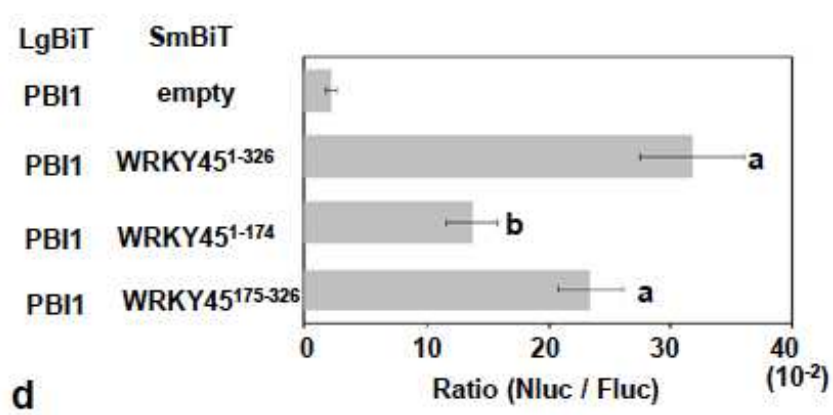

e
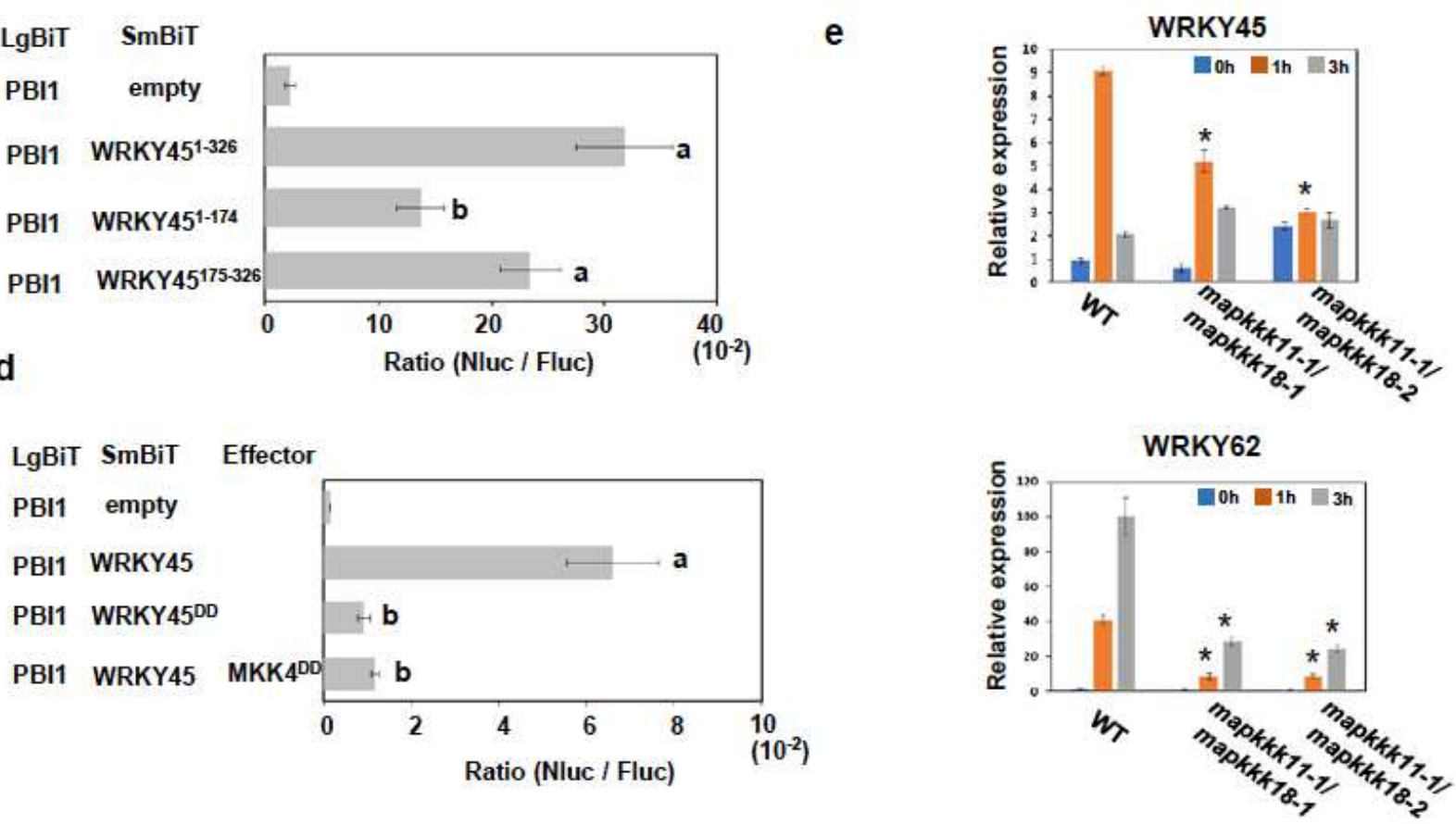

Figure 6 
MAPKs regulate PBI1 degradation. a, Chitin-induced MAPK activation in two mapkkk11/mapkkk18 mutants. Total proteins were prepared from rice suspension-cultured cells after treatment with $2 \mu \mathrm{g}$ (GluNAc)7 and subjected to immunoblots with a-pMAPK. b, Chitin-induced PBI1 degradation was inhibited in the mapkkk11/mapkkk18 mutants. Total proteins were prepared as for (a) and probed with aPBI1. c, The interactions between PBI1 and full length WRKY45 or WRKY45 fragments were analyzed using split NanoLuc assays. Constructs were made to produce PBI1 fused to LgBiT and the WRKY45 fragments fused to SmBiT. Fluc was used as an internal control. Rice protoplasts were transfected with the constructs and the interactions were indicated by the Nluc to Fluc ratios. Different letters above the data points indicate significant differences ( $p<0.01$, Welch's $t$ test). $d$, Phosphorylation of WRKY45 inhibits the interaction between PBI1 and WRKY45. Split NanoLuc assays were carried out by transient expression of PBI1- LgBiT and WRKY45-SmBiT or WRKY45DD-SmBiT with or without MKK4DD in rice protoplasts. Values are means \pm S.E. Different letters above the data points indicate significant differences ( $p<0.01$, Welch's $t$ test). e, The expression levels of WRKY45 and WRKY62 in mapkkk11/mapkkk18 suspension-cultured cells treated with $2 \mu \mathrm{g}$ (GluNAc)7 were analyzed using quantitative real-time PCR. Data are means $\pm S D$ from three independent biological replicates, where each biological replicate consisted of two technical replicates. The asterisks indicate statistically significant differences from the WT controls by Student's t-test $(P<0.05)$. 

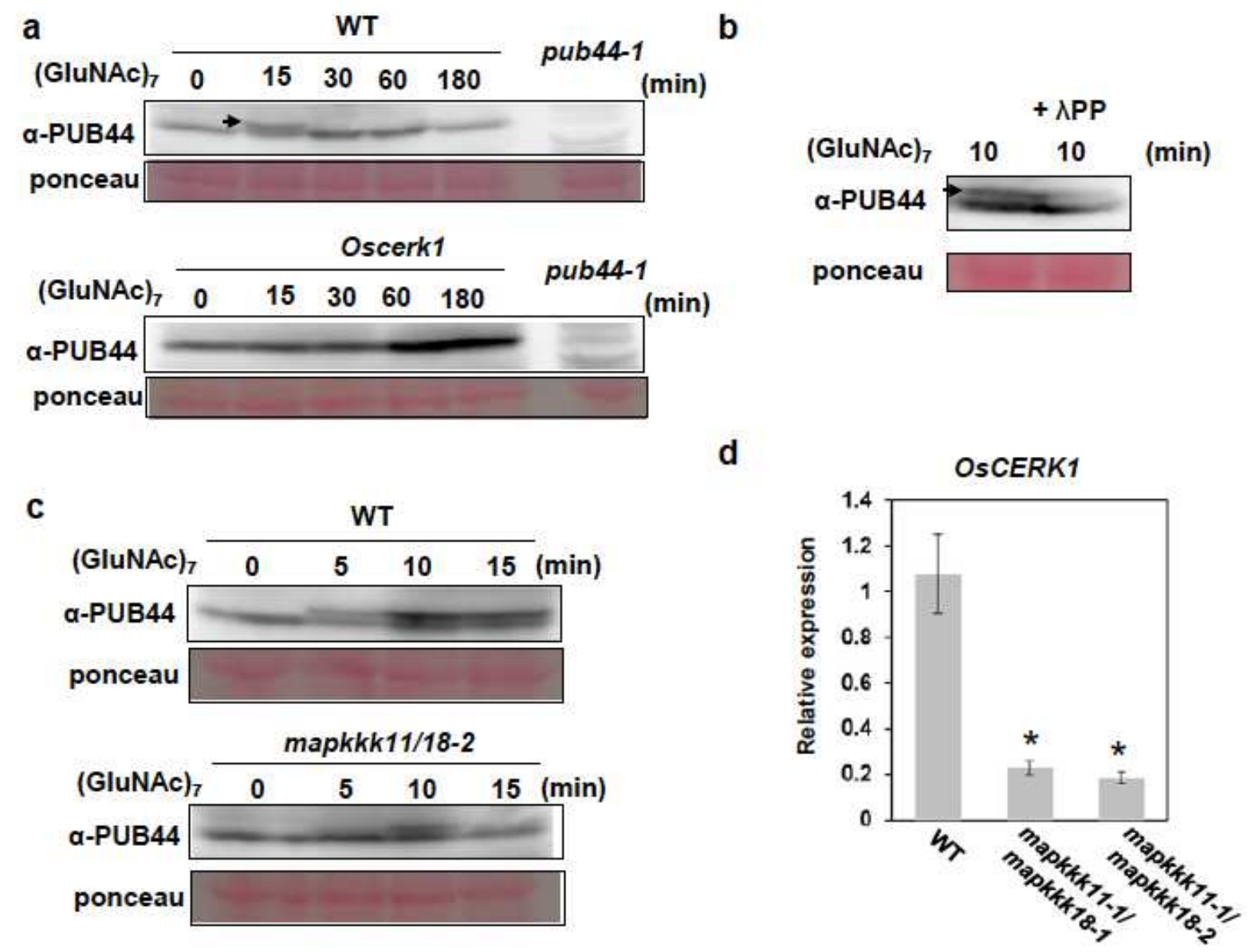

\section{Figure 7}

PUB44 is phosphorylated upon chitin perception. a, A mobility shift of PUB44 was detected by immunoblotting with a-PUB44 using total proteins prepared from WT rice suspension-cultured cells after treatment with $2 \mu \mathrm{g}$ (GluNAc)7 (upper panel). The arrow indicates the shifted PUB44 band. The mobility shift did not occur in Oscerk1 mutant cells (lower panel). b, The mobility shift of PUB44 was reversed by treatment with $\lambda$ protein phosphatase, indicating that the shift was due to phosphorylation of PUB44. The arrow indicates the shifted PUB44 band. c, PUB44 phosphorylation was delayed and reduced in the mapkkk11/18 mutant (lower panel) when compared with WT cells (upper panel). d, OsCERK1 transcript levels in WT and mapkkk11/18 mutant cells, measured by quantitative real-time PCR. Data are means $\pm S D$ from three independent biological replicates, where each biological replicate consisted of two technical replicates. The asterisks indicate statistically significant differences from the WT controls by Student's t-test $(P<0.05)$. 


\section{Supplementary Files}

This is a list of supplementary files associated with this preprint. Click to download.

- ExtendedData.pdf 